\title{
ON Lie-ISOCLINIC LEIBNIZ ALGEBRAS
}

\author{
G. R. Biyogmam ${ }^{(1)}$ and J. M. Casas ${ }^{(2)}$ \\ (1) Department of Mathematics, Southwestern Oklahoma State University \\ Weatherford, OK 73096, USA \\ E-mail address: guy.biyogmam@swosu.edu \\ ${ }^{(2)}$ Dpto. Matemática Aplicada, Universidad de Vigo, E. E. Forestal \\ Campus Universitario A Xunqueira, 36005 Pontevedra, Spain \\ E-mail address: jmcasas@uvigo.es
}

\begin{abstract}
In this paper we study the notion of isoclinism on Lie-central extensions of Leibniz algebras, this yields to introduce the concept of Lie-isoclinic Leibniz algebras. We provide several equivalent conditions under which Leibniz algebras are Lie-isoclinic. We also define the concept of Schur Lie-multiplier and analyze its connection with Lie-isoclinism.
\end{abstract}

2010 MSC: 17A32, 17B55, $18 B 99$.

Key words: Leibniz algebras, Lie-isoclinic extensions, Lie-Schur multiplier, Lie-homology.

\section{Introduction}

The concept of isoclinism goes back to Philip Hall [7] in 1940 in his attempt to classify $p$-groups using an equivalence relation weaker than the notion of isomorphism. This concept was extended to Lie algebras by K. Moneyhun in 14, and later by several other authors [12, 13, 15, 16, 17]. Our main purpose on this paper is to extend the study of isoclinism on non Lie-Leibniz algebras, relying on the anti-symmetry of their Leibniz bracket. Nevertheless, the generalization of isoclinism concepts from Lie to Leibniz algebras seems to be a mere adaptation of techniques and results, so our approach focuses in the relative notions of isoclinism.

Relative means that we are approaching the concepts relative to the Liezation functor $(-)_{\text {Lie }}:$ Leib $\rightarrow$ Lie which assigns to a Leibniz algebra $\mathfrak{q}$ the Lie algebra $\mathfrak{q}_{\text {Lie }}=\mathfrak{q} /<\{[x, x]: x \in \mathfrak{q}\}>$. With other words, from the categorical theory of central extensions relative to a chosen subcategory of a base category given in [8], the concepts of central extension, perfect object and commutator in a semi-abelian category relative to a Birkhoff subcategory were approached in [4]. In particular, in [2] was approached the case of central extensions of Leibniz algebras with respect to its Birkhoff subcategory of Lie algebras, that is, with 
respect to the Liezation functor. The absolute case in this theory corresponds to the abelianization functor which assigns to a Leibniz (Lie) algebra the abelian Leibniz (Lie) algebra with the same underlying vector space, but with trivial bracket operation. This absolute case corresponds to the classical case described at the beginning.

In this paper we continue the study of central extensions relative to the Liezation functor, so called Lie-central extensions, initiated in [2]. In concrete, we study isoclinism of Lie-central extensions of Leibniz algebras. To do so, we organize the paper as follows, in Section 2 we recall the Lie-notions defined in [2]. In Section 3, we define the notion of Lie-isoclinism between two Lie-central extensions of Leibniz algebras and provide necessary and sufficient conditions under which two Leibniz algebras are Lie-isoclinic. Also in [2], the authors defined the Lie-homology of Leibniz algebras which enables us to analyze the connection between Lie-isoclinism and the Schur Lie-multiplier in Section 4 . In this section, we provide another characterization of Lie-isoclinic Leibniz algebras by means of the Lie-homology of the isomorphisms between their Lie-centers and their Lie-commutators. This is used to determine the group of Lie-autoclinisms of any given Leibniz algebra. Finally we prove that under certain conditions, all Lie-stem covers of a given Leibniz algebra are mutually Lie-isoclinic.

\section{Preliminary results on Leibniz algebras}

We fix $\mathbb{K}$ as a ground field such that $\frac{1}{2} \in \mathbb{K}$. All vector spaces and tensor products are considered over $\mathbb{K}$.

A Leibniz algebra [10, 11] is a vector space $\mathfrak{q}$ equipped with a bilinear map $[-,-]: \mathfrak{q} \otimes \mathfrak{q} \rightarrow \mathfrak{q}$, usually called the Leibniz bracket of $\mathfrak{q}$, satisfying the Leibniz identity:

$$
[x,[y, z]]=[[x, y], z]-[[x, z], y], \quad x, y, z \in \mathfrak{q} .
$$

Leibniz algebras form a semi-abelian category [3, 9], denoted by Leib, whose morphisms are linear maps that preserve the Leibniz bracket.

A subalgebra $\mathfrak{h}$ of a Leibniz algebra $\mathfrak{q}$ is said to be left (resp. right) ideal of $\mathfrak{q}$ if $[h, q] \in \mathfrak{h}(\operatorname{resp} .[q, h] \in \mathfrak{h})$, for all $h \in \mathfrak{h}, q \in \mathfrak{q}$. If $\mathfrak{h}$ is both left and right ideal, then $\mathfrak{h}$ is called two-sided ideal of $\mathfrak{q}$. In this case $\mathfrak{q} / \mathfrak{h}$ naturally inherits a Leibniz algebra structure.

For a Leibniz algebra $\mathfrak{q}$, we denote by $\mathfrak{q}^{\text {ann }}$ the subspace of $\mathfrak{q}$ spanned by all elements of the form $[x, x], x \in \mathfrak{q}$.

Given a Leibniz algebra $\mathfrak{q}$, it is clear that the quotient $\mathfrak{q}_{\text {Lie }}=\mathfrak{q} / \mathfrak{q}^{\text {ann }}$ is a Lie algebra. This defines the so-called Liezation functor $(-)_{\text {Lie }}:$ Leib $\rightarrow$ Lie, which assigns to a Leibniz algebra $\mathfrak{q}$ the Lie algebra $\mathfrak{q}_{\text {Lie }}$. Moreover, the canonical epimorphism $\mathfrak{q} \rightarrow \mathfrak{q}_{\text {Lie }}$ is universal among all homomorphisms from $\mathfrak{q}$ to a Lie algebra, implying that the Liezation functor is left adjoint to the inclusion functor Lie $\hookrightarrow$ Leib. 
The following notions come from 2 .

Let $\mathfrak{q}$ be a Leibniz algebra and $\mathfrak{m}, \mathfrak{n}$ be two-sided ideals of $\mathfrak{q}$. The Liecommutator of $\mathfrak{m}$ and $\mathfrak{n}$ is the two-sided ideal of $\mathfrak{q}$

$$
[\mathfrak{m}, \mathfrak{n}]_{\text {Lie }}=\langle\{[m, n]+[n, m], m \in \mathfrak{m}, n \in \mathfrak{n}\}\rangle .
$$

The Lie-center of the Leibniz algebra $\mathfrak{q}$ is the two-sided-ideal

$$
Z_{\text {Lie }}(\mathfrak{q})=\{z \in \mathfrak{q} \mid[q, z]+[z, q]=0 \text { for all } q \in \mathfrak{q}\} .
$$

An extension of Leibniz algebras $0 \rightarrow \mathfrak{n} \rightarrow \mathfrak{g} \stackrel{\pi}{\rightarrow} \mathfrak{q} \rightarrow 0$ is said to be Lie-central if $\mathfrak{n} \subseteq Z_{\text {Lie }}(\mathfrak{q})$, equivalently, $[\mathfrak{n}, \mathfrak{g}]_{\text {Lie }}=0$.

Following [2, 4, given a free presentation $0 \rightarrow \mathfrak{r} \rightarrow \mathfrak{f} \stackrel{\rho}{\rightarrow} \mathfrak{g} \rightarrow 0$ of Leibniz algebra $\mathfrak{g}$, the second Lie-homology with trivial coefficients is given by

$$
\mathrm{HL}_{2}^{\text {Lie }}(\mathfrak{g}) \cong \frac{\mathfrak{r} \cap[\mathfrak{f}, \mathfrak{f}]_{\text {Lie }}}{[\mathfrak{r}, \mathfrak{f}]_{\text {Lie }}}
$$

On the other hand, the first Lie-homology with trivial coefficients is given by $\mathrm{HL}_{1}^{\text {Lie }}(\mathfrak{g}) \cong \mathfrak{g}_{\text {Lie }}$.

\section{Lie-isoclinic Leibniz algebras}

Consider the Lie-central extensions $(g): 0 \rightarrow \mathfrak{n} \stackrel{\chi}{\rightarrow} \mathfrak{g} \stackrel{\pi}{\rightarrow} \mathfrak{q} \rightarrow 0$ and $\left(g_{i}\right): 0 \rightarrow$ $\mathfrak{n}_{i} \stackrel{\chi_{i}}{\rightarrow} \mathfrak{g}_{i} \stackrel{\pi_{i}}{\rightarrow} \mathfrak{q}_{i} \rightarrow 0, i=1,2$,

Let be $C: \mathfrak{q} \times \mathfrak{q} \rightarrow[\mathfrak{g}, \mathfrak{g}]_{\text {Lie }}$ given by $C\left(q_{1}, q_{2}\right)=\left[g_{1}, g_{2}\right]+\left[g_{2}, g_{1}\right]$, where $\pi\left(g_{j}\right)=q_{j}, j=1,2$, the Lie-commutator map associated to the extension $(g)$. In a similar way is defined the Lie-commutator map $C_{i}$ corresponding to the extensions $\left(g_{i}\right), i=1,2$.

Note that if $\mathfrak{q}$ is a Lie algebra, then $\pi\left([\mathfrak{g}, \mathfrak{g}]_{\text {Lie }}\right)=0$, hence $[\mathfrak{g}, \mathfrak{g}]_{\text {Lie }} \subseteq \mathfrak{n} \equiv \chi(\mathfrak{n})$.

Definition 3.1 The Lie-central extensions $\left(g_{1}\right)$ and $\left(g_{2}\right)$ are said to be Lie-isoclinic when there exist isomorphisms $\eta: \mathfrak{q}_{1} \rightarrow \mathfrak{q}_{2}$ and $\xi:\left[\mathfrak{g}_{1}, \mathfrak{g}_{1}\right]_{\text {Lie }} \rightarrow\left[\mathfrak{g}_{2}, \mathfrak{g}_{2}\right]_{\text {Lie }}$ such that the following diagram is commutative:

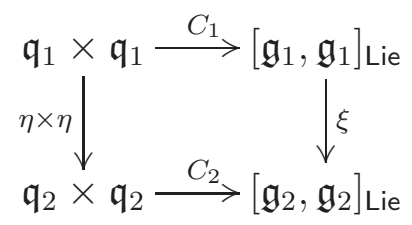

The pair $(\eta, \xi)$ is called a Lie-isoclinism from $\left(g_{1}\right)$ to $\left(g_{2}\right)$ and will be denoted by $(\eta, \xi):\left(g_{1}\right) \rightarrow\left(g_{2}\right)$.

Remark 3.2 In fact it suffices to assume in Definition 3.1 that $\xi$ is a monomorphism. Furthermore, $\eta$ determines $\xi$ uniquely. 
Let $\mathfrak{q}$ be Leibniz algebra, then we can construct the following Lie-central extension

$$
\left(e_{q}\right): 0 \rightarrow Z_{\text {Lie }}(\mathfrak{q}) \rightarrow \mathfrak{q} \stackrel{p r_{\mathfrak{q}}}{\rightarrow} \mathfrak{q} / Z_{\text {Lie }}(\mathfrak{q}) \rightarrow 0
$$

Definition 3.3 Let $\mathfrak{g}$ and $\mathfrak{q}$ be Leibniz algebras. Then $\mathfrak{g}$ and $\mathfrak{q}$ are said to be Lie-isoclinic when $\left(e_{g}\right)$ and $\left(e_{q}\right)$ are Lie-isoclinic Lie-central extensions.

A Lie-isoclinism $(\eta, \xi)$ from $\left(e_{g}\right)$ to $\left(e_{q}\right)$ is also called a Lie-isoclinism from $\mathfrak{g}$ to $\mathfrak{q}$, denoted by $(\eta, \xi): \mathfrak{g} \sim \mathfrak{q}$.

Proposition 3.4 For a Lie-isoclinism $(\eta, \xi):\left(g_{1}\right) \sim\left(g_{2}\right)$, the following statements hold:

a) $\eta$ induces an isomorphism $\eta^{\prime}: \mathfrak{g}_{1} / Z_{\text {Lie }}\left(\mathfrak{g}_{1}\right) \rightarrow \mathfrak{g}_{2} / Z_{\text {Lie }}\left(\mathfrak{g}_{2}\right)$, and $\left(\eta^{\prime}, \xi\right)$ is a Lie-isoclinism from $\mathfrak{g}_{1}$ to $\mathfrak{g}_{2}$.

b) $\chi_{1}\left(\mathfrak{n}_{1}\right)=Z_{\text {Lie }}\left(\mathfrak{g}_{1}\right)$ if and only if $\chi_{2}\left(\mathfrak{n}_{2}\right)=Z_{\text {Lie }}\left(\mathfrak{g}_{2}\right)$.

Proof. Let $(\eta, \xi):\left(g_{1}\right) \sim\left(g_{2}\right)$ be a Lie-isoclinism. Then consider the map $\phi: \mathfrak{g}_{1} \rightarrow \mathfrak{g}_{2} / Z_{\text {Lie }}\left(\mathfrak{g}_{2}\right)$ defined by $g_{1} \mapsto \bar{g}_{2}$ where $\pi_{2}\left(g_{2}\right)=\left(\eta \circ \pi_{1}\right)\left(g_{1}\right)$. Clearly, $\phi$ is a well-defined onto homomorphism since $\left(g_{2}\right)$ is a Lie-central extension. We claim that $\operatorname{Ker}(\phi)=Z_{\text {Lie }}\left(\mathfrak{g}_{1}\right)$. Indeed, Let $g_{1} \in Z_{\text {Lie }}\left(\mathfrak{g}_{1}\right)$ and let $y \in \mathfrak{g}_{2}$. Then set $\bar{g}_{2}:=\phi\left(g_{1}\right)$ with $g_{2} \in \mathfrak{g}_{2}$. Now since $\phi$ is onto, $\bar{y}=\phi(x)$ for some $x \in \mathfrak{g}_{1}$. Then by commutativity of the diagram induced by the Lie-isoclinism $(\eta, \xi)$,

$$
\begin{aligned}
{\left[g_{2}, y\right]+\left[y, g_{2}\right] } & =C_{2}\left(\pi_{2}\left(g_{2}\right), \pi_{2}(y)\right) \\
& =C_{2}\left(\eta\left(\pi_{1}\left(g_{1}\right)\right), \eta\left(\pi_{1}(x)\right)\right) \\
& =C_{2}\left(\eta \times \eta\left(\pi_{1}\left(g_{1}\right), \pi_{1}(x)\right)\right) \\
& =\xi\left(C_{1}\left(\pi_{1}\left(g_{1}\right), \pi_{1}(x)\right)\right) \\
& =\xi\left(\left[g_{1}, x\right]+\left[x, g_{1}\right]\right) \\
& =\xi(0) \\
& =0 .
\end{aligned}
$$

So $g_{2} \in Z_{\text {Lie }}\left(\mathfrak{g}_{2}\right)$. Therefore $g_{1} \in \operatorname{Ker}(\phi)$.

Conversely, let $g_{1} \in \operatorname{Ker}(\phi)$ i.e. $\bar{g}_{2}:=\phi\left(g_{1}\right)=0$ with $g_{2} \in Z_{\text {Lie }}\left(\mathfrak{g}_{2}\right)$. For every $x \in \mathfrak{g}_{1},\left(\eta \circ \pi_{1}\right)(x) \in \mathfrak{q}_{2}$. As $\pi_{2}$ is onto, $\left(\eta \circ \pi_{1}\right)(x)=\pi_{2}(y)$ for some $y \in \mathfrak{g}_{2}$. Again by commutativity of the diagram induced by the Lie-isoclinism $(\eta, \xi)$,

$$
\begin{aligned}
\xi\left(C_{1}\left(\pi_{1}\left(g_{1}\right), \pi_{1}(x)\right)\right) & =C_{2}\left(\eta\left(\pi_{1}\left(g_{1}\right)\right), \eta\left(\left(\pi_{1}(x)\right)\right)\right. \\
& =C_{2}\left(\pi_{2}\left(g_{2}\right), \pi_{2}(y)\right) \\
& =\left[g_{2}, y\right]+\left[y, g_{2}\right] \\
& =0 .
\end{aligned}
$$

So $\left[g_{1}, x\right]+\left[x, g_{1}\right]=C_{1}\left(\pi_{1}\left(g_{1}\right), \pi_{1}(x)\right) \in \operatorname{Ker}(\xi)=0$. Thus $\left[g_{1}, x\right]+\left[x, g_{1}\right]=0$. Hence $g_{1} \in Z_{\text {Lie }}\left(\mathfrak{g}_{1}\right)$. $\eta^{\prime}$ is obtained by the first isomorphism theorem and an easy checking shows that $\eta^{\prime}$ is an isomorphism. 
It remains to show that the following diagram is commutative:

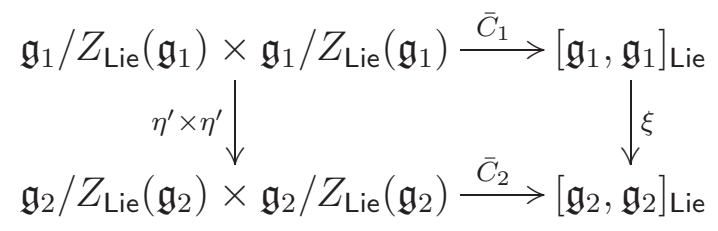

Indeed, since $\mathfrak{n}_{i} \subseteq Z_{\text {Lie }}\left(\mathfrak{q}_{i}\right)$ for $i=1,2$, we have well-defined maps $P_{i}: \mathfrak{q}_{i} \rightarrow$ $\mathfrak{g}_{i} / Z_{\text {Lie }}\left(\mathfrak{g}_{i}\right), z^{i} \mapsto \bar{x}^{i}$ where $z^{i}=\pi_{i}\left(x^{i}\right)$. These maps induce the well-defined maps $\bar{C}_{i}: \mathfrak{g}_{i} / Z_{\text {Lie }}\left(\mathfrak{g}_{i}\right) \times \mathfrak{g}_{i} / Z_{\text {Lie }}\left(\mathfrak{g}_{i}\right) \rightarrow\left[\mathfrak{g}_{i}, \mathfrak{g}_{i}\right]_{\text {Lie }}$ defined by $\bar{C}_{i}\left(\bar{x}_{1}^{i}, \bar{x}_{2}^{i}\right):=C_{1}\left(z_{1}^{i}, z_{2}^{i}\right)$. Now let $g_{1}, g_{2} \in \mathfrak{g}_{1}$ such that $\pi_{1}\left(g_{1}\right)=q_{1}$ and $\pi_{2}\left(g_{2}\right)=q_{2}$, and let $h_{1}, h_{2} \in \mathfrak{g}_{2}$ such that $\pi_{2}\left(h_{1}\right)=\left(\eta \circ \pi_{1}\right)\left(g_{1}\right)$ and $\pi_{2}\left(h_{2}\right)=\left(\eta \circ \pi_{1}\right)\left(g_{2}\right)$. Then

$$
\begin{aligned}
\xi \circ \bar{C}_{1}\left(\bar{g}_{1}, \bar{g}_{2}\right) & \left.=\xi \circ C_{1}\left(q_{1}, q_{2}\right)=\left(C_{2} \circ(\eta \times \eta)\left(q_{1}, q_{2}\right)\right)\right) \\
& =C_{2}\left(\left(\eta \circ \pi_{1}\right)\left(g_{1}\right),\left(\eta \circ \pi_{1}\right)\left(g_{2}\right)\right) \\
& =C_{2}\left(\pi_{2}\left(h_{1}\right), \pi_{2}\left(h_{2}\right)\right) \\
& =\bar{C}_{2}\left(\bar{h}_{1}, \bar{h}_{2}\right) \\
& =\bar{C}_{2}\left(\eta^{\prime}\left(\bar{g}_{1}\right), \eta^{\prime}\left(\bar{g}_{2}\right)\right) \\
& =\left(\bar{C}_{2} \circ\left(\eta^{\prime} \times \eta^{\prime}\right)\right)\left(\bar{g}_{1}, \bar{g}_{2}\right) .
\end{aligned}
$$

Therefore the diagram above is commutative.

To show b), assume that $\chi_{1}\left(\mathfrak{n}_{1}\right)=Z_{\text {Lie }}\left(\mathfrak{g}_{1}\right)$ and let $z \in \chi_{2}\left(\mathfrak{n}_{2}\right)$ i.e. $\pi_{2}(z)=0$. Since $\eta^{\prime}$ is onto, $\eta^{\prime}\left(\bar{z}^{\prime}\right)=\bar{z}$ for some $z^{\prime} \in \mathfrak{g}_{1}$. Then $\left(\eta \circ \pi_{1}\right)\left(z^{\prime}\right)=\pi_{2}(z)=0$ i.e. $\eta\left(\pi_{1}\left(z^{\prime}\right)\right)=0$. This implies that $\pi_{1}\left(z^{\prime}\right)=0$ since $\eta$ is one to one. So $z^{\prime} \in \chi_{1}\left(\mathfrak{n}_{1}\right)=$ $Z_{\text {Lie }}\left(\mathfrak{g}_{1}\right)$, and thus $\bar{z}=\eta^{\prime}\left(\bar{z}^{\prime}\right)=0$ in $\mathfrak{g}_{2} / Z_{\text {Lie }}\left(\mathfrak{g}_{2}\right)$. Hence $z \in Z_{\text {Lie }}\left(\mathfrak{g}_{2}\right)$. Conversely, let $z \in Z_{\text {Lie }}\left(\mathfrak{g}_{2}\right)$. Then $\bar{z}=0$ in $\mathfrak{g}_{2} / Z_{\text {Lie }}\left(\mathfrak{g}_{2}\right)$. Since $\eta^{\prime}$ is an isomorphism, $\eta^{\prime}(\bar{t})=0$ for some $t \in Z_{\text {Lie }}\left(\mathfrak{g}_{1}\right)=\chi_{1}\left(\mathfrak{n}_{1}\right)$. So $\pi_{2}(z)=\left(\eta \circ \pi_{1}\right)(t)=\eta\left(\pi_{1}(t)\right)=\eta(0)=0$. Therefore $z \in \chi_{2}\left(\mathfrak{n}_{2}\right)$.

The other implication is identical.

Proposition 3.5 Lie-isoclinism is an equivalence relation.

Proof. Direct cheking.

Lie-isoclinisms of the form $(\eta, \xi):(g) \sim(g)$, respectively $(\eta, \xi): \mathfrak{g} \sim \mathfrak{g}$, are called autoclinisms of $(g)$, respectively $\mathfrak{g}$, and they constitute the group $\operatorname{Autcl}(\mathrm{g})$, respectively $\operatorname{Autcl}(\mathfrak{g})$. Each Lie-isoclinism from $\left(g_{1}\right)$ to $\left(g_{2}\right)$ induces an isomorphism from Autcl $\left(g_{1}\right)$ to $\operatorname{Autcl}\left(g_{2}\right)$.

Let $(\alpha, \beta, \gamma):\left(g_{1}\right) \rightarrow\left(g_{2}\right)$ be an isomorphism of Lie-central extensions (i.e. $\alpha, \beta, \gamma$ are bijective Leibniz homomorphisms such that $\chi_{2} \circ \alpha=\beta \circ \chi_{1}$ and $\pi_{2} \circ \beta=$ $\left.\gamma \circ \pi_{1}\right)$ and $\beta^{\prime}$ the restriction of $\beta$ to $\left[\mathfrak{g}_{1}, \mathfrak{g}_{1}\right]_{\text {Lie }}$. Then $\left(\gamma, \beta^{\prime}\right)$ is a Lie-isoclinism from $\left(g_{1}\right)$ to $\left(g_{2}\right)$. 
From Proposition 3.4, the classes of Lie-isoclinic Leibniz algebras can be regarded as those isoclinism classes that consist of Lie-central extensions $(g)$ with $\chi(\mathfrak{n})=Z_{\text {Lie }}(\mathfrak{g})$, i. e. $(g)$ is isomorphic to $\left(e_{g}\right)$.

The Leibniz algebras which are Lie-isoclinic to the trivial Leibniz algebra are exactly the abelian ones. Indeed, if $\mathfrak{g}_{1}$ is a Leibniz algebra and $\mathfrak{g}_{2}$ the trivial Leibniz algebra which are Lie-isoclinic, then the isomorphism $\xi:\left[\mathfrak{g}_{1}, \mathfrak{g}_{1}\right]_{\text {Lie }} \rightarrow$ $\left[\mathfrak{g}_{2}, \mathfrak{g}_{2}\right]_{\text {Lie }}$ and the fact $\left[\mathfrak{g}_{2}, \mathfrak{g}_{2}\right]_{\text {Lie }}=0$ implies that $\left[\mathfrak{g}_{1}, \mathfrak{g}_{1}\right]_{\text {Lie }}=0$, so the isomorphism $\eta: \mathfrak{g}_{1} \rightarrow \mathfrak{g}_{2}$ implies that $\mathfrak{g}_{1}$ is an abelian Leibniz algebra.

Conversely, every abelian Leibniz algebra $\mathfrak{g}$ is Lie-isoclinic to the trivial Leibniz algebra on the underlying set $\mathfrak{g}$. Indeed, for both Leibniz algebras, $Z_{\text {Lie }}(\mathfrak{g})=\mathfrak{g}$ and thus $\mathfrak{g} / Z_{\text {Lie }}(\mathfrak{g})=0$. We then have the Lie- isoclinism $\left(\eta=i d_{\mathfrak{g}}, \xi=0\right):(\mathfrak{g}) \sim(\mathfrak{g})$.

Example 3.6 Following we provide an example of two non-abelian Lie-isoclinic Leibniz algebras.

Consider the non-abelian complex Leibniz algebras $\mathfrak{g}_{1}=\operatorname{span}\left\{e_{1}, e_{2}\right\}$ with nonzero multiplications $\left[e_{1}, e_{1}\right]=\left[e_{2}, e_{1}\right]=e_{2}$ (it belongs to the third isomorphism class of Lemma 2 in [5]), and $\mathfrak{g}_{2}=\operatorname{span}\left\{a_{1}, a_{2}, a_{3}\right\}$ with non-zero multiplications $\left[a_{1}, a_{1}\right]=\left[a_{2}, a_{1}\right]=\left[a_{3}, a_{1}\right]=a_{3}$ (it belongs to the isomorphism class 2 (d) of the classification given in [1]]). Clearly $\left[\mathfrak{g}_{1}, \mathfrak{g}_{1}\right]_{\mathrm{Lie}}=\operatorname{span}\left\{e_{2}\right\},\left[\mathfrak{g}_{2}, \mathfrak{g}_{2}\right]_{\mathrm{Lie}}=\operatorname{span}\left\{a_{3}\right\}$, $Z_{\text {Lie }}\left(\mathfrak{g}_{1}\right)=0$ and $Z_{\text {Lie }}\left(\mathfrak{g}_{2}\right)=\operatorname{span}\left\{a_{2}-a_{3}\right\}$. So $\mathfrak{q}_{1}:=\mathfrak{g}_{1} / Z_{\text {Lie }}\left(\mathfrak{g}_{1}\right) \simeq \mathfrak{g}_{1}$. Also applying the first isomorphism theorem on the map $\phi: \mathfrak{g}_{2} \rightarrow \mathfrak{g}_{2}$ defined by $\phi\left(a_{1}\right)=$ $a_{1}$ and $\phi\left(a_{2}\right)=\phi\left(a_{3}\right)=a_{3}$ shows $\mathfrak{q}_{2}:=\mathfrak{g}_{2} / Z_{\text {Lie }}\left(\mathfrak{g}_{2}\right) \simeq \operatorname{span}\left\{a_{1}, a_{3}\right\}$. Now define $\eta: \mathfrak{q}_{1} \rightarrow \mathfrak{q}_{2}$ by $\eta\left(e_{1}\right)=a_{1}$ and $\eta\left(e_{2}\right)=a_{3}$, and $\xi:\left[\mathfrak{g}_{1}, \mathfrak{g}_{1}\right]_{\text {Lie }} \rightarrow\left[\mathfrak{g}_{2}, \mathfrak{g}_{2}\right]_{\text {Lie }}$ by $\xi\left(e_{2}\right)=a_{3}$. It is easy to show that $(\eta, \xi):(\mathfrak{g}) \sim(\mathfrak{g})$ is a Lie-isoclinism.

Proposition 3.7 Let $(\eta, \xi):\left(g_{1}\right) \sim\left(g_{2}\right)$ be a Lie-isoclinism. Then the backward induced extension $\left(\eta^{*}\left(g_{2}\right)\right): 0 \rightarrow \mathfrak{n}_{2} \rightarrow \mathfrak{g}_{2}^{\eta} \stackrel{\bar{\pi}_{2}}{\rightarrow} \mathfrak{q}_{1} \rightarrow 0$, obtained by pulling back along $\eta$ (where $\mathfrak{g}_{2}^{\eta}=\left\{(g, q) \in \mathfrak{g}_{2} \times \mathfrak{q}_{1} \mid \pi_{2}(g)=\eta(q)\right\}$ ) is a Lie-central extension isomorphic to $\left(g_{2}\right)$, and $\left(i d_{\mathfrak{q}_{1}}, \xi\right):\left(g_{1}\right) \sim\left(\eta^{*}\left(g_{2}\right)\right)$ is a Lie-isoclinism.

Proof. The first statement only requires a routine checking.

To show that $\left(i d_{\mathfrak{q}_{1}}, \xi\right):\left(g_{1}\right) \sim\left(\eta^{*} g_{2}\right)$ is a Lie-isoclinism, have in mind that $\xi$ induces an isomorphism (call it also $\xi) \xi:\left[\mathfrak{g}_{1}, \mathfrak{g}_{1}\right]_{\text {Lie }} \rightarrow\left[\mathfrak{g}_{2}^{\eta}, \mathfrak{g}_{2}^{\eta}\right]_{\text {Lie }}$ defined by $\xi\left(\left[g_{1}, g_{1}^{\prime}\right]+\left[g_{1}^{\prime}, g_{1}\right]\right)=\left(\left[g_{2}, g_{2}^{\prime}\right]+\left[g_{2}^{\prime}, g_{2}\right],\left[q_{1}, q_{1}^{\prime}\right]+\left[q_{1}^{\prime}, q_{1}\right]\right)$, where $\pi_{2}\left(g_{2}\right)=\eta\left(q_{1}\right)=$ $\eta \pi_{1}\left(g_{1}\right)$. The commutativity of the diagram



where $C_{2}^{\eta}\left(q_{1}, q_{1}^{\prime}\right)=\left(\left[g_{2}, g_{2}^{\prime}\right]+\left[g_{2}^{\prime}, g_{2}\right],\left[q_{1}, q_{1}^{\prime}\right]+\left[q_{1}^{\prime}, q_{1}\right]\right)$, follows directly.

Thanks to Proposition 3.7, in many cases we can restrict ourselves to Lieisoclinisms $(\eta, \xi)$ in which $\eta$ is an identity map. 
Proposition 3.8 Let $(\eta, \xi):\left(g_{1}\right) \sim\left(g_{2}\right)$ be an Lie-isoclinism. Then the following statements hold:

a) $\pi_{2} \circ \xi(g)=\eta \circ \pi_{1}(g)$, for all $g \in\left[\mathfrak{g}_{1}, \mathfrak{g}_{1}\right]_{\text {Lie }}$.

b) $\xi\left(\chi_{1}\left(\mathfrak{n}_{1}\right) \cap\left[\mathfrak{g}_{1}, \mathfrak{g}_{1}\right]_{\text {Lie }}\right)=\chi_{2}\left(\mathfrak{n}_{2}\right) \cap\left[\mathfrak{g}_{2}, \mathfrak{g}_{2}\right]_{\text {Lie }}$.

c) $\xi([g, x]+[x, g])=[h, \xi(x)]+[\xi(x), h]$, for all $x \in\left[\mathfrak{g}_{1}, \mathfrak{g}_{1}\right]_{\text {Lie }}, g \in \mathfrak{g}_{1}, h \in \mathfrak{g}_{2}$ such that $\eta \circ \pi_{1}(g)=\pi_{2}(h)$.

Proof. a) Proposition 3.7 establishes that the Lie-isoclinism $(\eta, \xi):\left(g_{1}\right) \sim\left(g_{2}\right)$ induces a Lie-isoclinism $\left(i d_{\mathfrak{q}_{1}}, \xi\right):\left(g_{1}\right) \sim\left(\eta^{*} g_{2}\right)$ where $\left(\xi(g), \pi_{1}(g)\right) \in\left[\mathfrak{g}_{2}^{\eta}, \mathfrak{g}_{2}^{\eta}\right]$ Lie for all $g \in\left[\mathfrak{g}_{1}, \mathfrak{g}_{1}\right]_{\text {Lie }} \subseteq \mathfrak{g}_{2}^{\eta}$.

b) Let $g \in \chi_{1}\left(\mathfrak{n}_{1}\right) \cap\left[\mathfrak{g}_{1}, \mathfrak{g}_{1}\right]_{\text {Lie }}$, then $\pi_{1}(g)=0$ which implies by a) that $\pi_{2}(\xi(g))=0$. So $\xi(g) \in \operatorname{Ker}\left(\pi_{2}\right)=\chi_{2}\left(\mathfrak{n}_{2}\right)$.

Conversely, let $h \in \chi_{2}\left(\mathfrak{n}_{2}\right) \cap\left[\mathfrak{g}_{2}, \mathfrak{g}_{2}\right]_{\text {Lie }}$. Then $\pi_{2}(h)=0$ and $h=\xi(g)$ for some $g \in\left[\mathfrak{g}_{1}, \mathfrak{g}_{1}\right]_{\text {Lie }}$ since $\xi$ is onto. Again by a) we have $\eta \circ \pi_{1}(g)=0$. This implies that $\pi_{1}(g)=0$. Thus $g \in \chi_{1}\left(\mathfrak{n}_{1}\right)$.

c) Let $x \in\left[\mathfrak{g}_{1}, \mathfrak{g}_{1}\right]_{\text {Lie }}, g \in \mathfrak{g}_{1}$ and $h \in \mathfrak{g}_{2}$ such that $\eta\left(\pi_{1}(g)\right)=\pi_{2}(h)$. Then

$$
\begin{aligned}
\xi([g, x]+[x, g]) & =\xi\left(C_{1}\left(\pi_{1}(g), \pi_{1}(x)\right)\right) \\
& =C_{2}\left(\eta \circ \pi_{1}(g), \eta \circ \pi_{1}(x)\right) \\
& =C_{2}\left(\pi_{2}(h), \pi_{2}(\xi(x))\right. \\
& =[h, \xi(x)]+[\xi(x), h] .
\end{aligned}
$$

As we have mentioned above, isomorphisms of Lie-central extensions induce Lie-isoclinisms. This observation gives rise to the following:

Definition 3.9 A homomorphism of Lie-central extensions $(\alpha, \beta, \gamma):\left(g_{1}\right) \rightarrow\left(g_{2}\right)$ is said to be Lie-isoclinic, if there exists an isomorphism $\beta^{\prime}:\left[\mathfrak{g}_{1}, \mathfrak{g}_{1}\right]_{\text {Lie }} \rightarrow\left[\mathfrak{g}_{2}, \mathfrak{g}_{2}\right]_{\text {Lie }}$ with $\left(\gamma, \beta^{\prime}\right):\left(g_{1}\right) \sim\left(g_{2}\right)$.

If $\beta$ is in addition an epimorphism (resp., monomorphism), then $(\alpha, \beta, \gamma)$ is called an isoclinic epimorphism (resp., monomorphism).

Proposition 3.10 For a homomorphism of Lie-central extensions $(\alpha, \beta, \gamma):\left(g_{1}\right) \rightarrow$ $\left(g_{2}\right)$, the following statements hold:

a) $(\alpha, \beta, \gamma)$ is Lie-isoclinic if and only if $\gamma$ is an isomorphism and $\operatorname{Ker}(\beta) \cap$ $\left[\mathfrak{g}_{1}, \mathfrak{g}_{1}\right]_{\text {Lie }}=0$.

b) If $(\alpha, \beta, \gamma)$ is Lie-isoclinic and $\beta^{\prime}$ as in Definition [3.9, then $\beta^{\prime}=\beta_{\left[\left[\mathfrak{g}_{1}, \mathfrak{g}_{1}\right]\right.}$ Lie. 
Proof. a) Assume that $(\alpha, \beta, \gamma):\left(\mathfrak{g}_{1}\right) \rightarrow\left(\mathfrak{g}_{2}\right)$ is Lie-isoclinic, then $\left(\gamma, \beta^{\prime}\right):\left(\mathfrak{g}_{1}\right) \sim$ $\left(\mathfrak{g}_{2}\right)$ is a Lie-isoclinism for some isomorphism $\beta^{\prime}:\left[\mathfrak{g}_{1}, \mathfrak{g}_{1}\right]_{\text {Lie }} \rightarrow\left[\mathfrak{g}_{2}, \mathfrak{g}_{2}\right]_{\text {Lie }}$. This implies by definition that $\gamma$ is an isomorphism. Now let $m \in \operatorname{Ker}(\beta) \cap\left[\mathfrak{g}_{1}, \mathfrak{g}_{1}\right]_{\text {Lie }}$. Then $\beta(m)=0$ and $m=\left[g_{1}, g_{2}\right]+\left[g_{2}, g_{1}\right]$ for some $g_{1}, g_{2} \in \mathfrak{g}_{1}$. Also let $q_{1}=\pi_{1}\left(g_{1}\right)$ and $q_{2}=\pi_{1}\left(g_{2}\right)$. Since $\left(\gamma, \beta^{\prime}\right):\left(\mathfrak{g}_{1}\right) \sim\left(\mathfrak{g}_{2}\right)$ is a Lie-isoclinism, we have

$$
\begin{aligned}
\beta^{\prime}(m) & =\beta^{\prime}\left(\left[g_{1}, g_{2}\right]+\left[g_{2}, g_{1}\right]\right) \\
& =\beta^{\prime}\left(C_{1}\left(\pi_{1}\left(g_{1}\right), \pi_{1}\left(g_{2}\right)\right)\right) \\
& =\left(\beta^{\prime} \circ C_{1}\right)\left(\pi_{1}\left(g_{1}\right), \pi_{1}\left(g_{2}\right)\right) \\
& =\left(C_{2} \circ(\gamma \times \gamma)\right)\left(\left(\pi_{1}\left(g_{1}\right), \pi_{1}\left(g_{2}\right)\right)\right. \\
& =C_{2}\left(\gamma\left(\pi_{1}\left(g_{1}\right)\right), \gamma\left(\pi_{1}\left(g_{2}\right)\right)\right) \\
& =C_{2}\left(\pi_{2}\left(\beta\left(g_{1}\right)\right), \pi_{2}\left(\beta\left(g_{2}\right)\right)\right) \\
& =\left[\beta\left(g_{1}\right), \beta\left(g_{2}\right)\right]+\left[\beta\left(g_{2}\right), \beta\left(g_{1}\right)\right] \\
& =\beta\left(\left[g_{1}, g_{2}\right]+\left[g_{2}, g_{1}\right]\right) \\
& =\beta(m)=0 .
\end{aligned}
$$

Since $\beta^{\prime}$ is one-to-one, it follows that $m=0$.

Conversely, assume that $\operatorname{Ker}(\beta) \cap\left[\mathfrak{g}_{1}, \mathfrak{g}_{1}\right]_{\text {Lie }}=0$. Define $\beta^{\prime}:\left[\mathfrak{g}_{1}, \mathfrak{g}_{1}\right]_{\text {Lie }} \rightarrow$ $\left[\mathfrak{g}_{2}, \mathfrak{g}_{2}\right]_{\text {Lie }}$ by $\beta^{\prime}(g)=\beta(g)$, which is one-to-one. It remains to show that $\beta^{\prime}$ is onto. Let $y \in\left[\mathfrak{g}_{2}, \mathfrak{g}_{2}\right]_{\text {Lie. }}$. Then $y=\left[g_{2}, g_{2}^{\prime}\right]+\left[g_{2}^{\prime}, g_{2}\right]$ for some $g_{2}, g_{2}^{\prime} \in \mathfrak{g}_{2}$. Since $\pi_{1}$ and $\gamma_{1}$ are onto, it follows that $\pi_{2}\left(g_{2}\right)=\left(\gamma \circ \pi_{1}\right)\left(g_{1}\right)$ and $\pi_{2}\left(g_{2}^{\prime}\right)=\left(\gamma \circ \pi_{1}\right)\left(g_{1}^{\prime}\right)$ for some $g_{1}, g_{1}^{\prime} \in \mathfrak{g}_{1}$. By the homomorphism $(\alpha, \beta, \gamma)$, we have $\left(\gamma \circ \pi_{1}\right)\left(g_{1}\right)=\left(\pi_{2} \circ \beta\right)\left(g_{1}\right)$ and $\left(\gamma \circ \pi_{1}\right)\left(g_{1}^{\prime}\right)=\left(\pi_{2} \circ \beta\right)\left(g_{1}^{\prime}\right)$, which implies that $g_{2}-\beta\left(g_{1}\right)=\chi_{2}(n)$ and $g_{2}^{\prime}-\beta\left(g_{1}^{\prime}\right)=\chi_{2}\left(n^{\prime}\right)$ for some $n, n^{\prime} \in \mathfrak{n}_{2}$. We now have

$$
\begin{aligned}
\beta^{\prime}\left(\left[g_{1}, g_{1}^{\prime}\right]+\left[g_{1}^{\prime}, g_{1}\right]\right)= & \beta\left(\left[g_{1}, g_{1}^{\prime}\right]+\left[g_{1}^{\prime}, g_{1}\right]\right) \\
= & {\left[\beta\left(g_{1}\right), \beta\left(g_{1}^{\prime}\right)\right]+\left[\beta\left(g_{1}^{\prime}\right), \beta\left(g_{1}\right)\right] } \\
= & {\left[g_{2}-\chi_{2}(n), g_{2}^{\prime}-\chi_{2}\left(n^{\prime}\right)\right]+\left[g_{2}^{\prime}-\chi_{2}\left(n^{\prime}\right), g_{2}-\chi_{2}(n)\right] } \\
= & y-\left(\left[\chi_{2}(n), g_{2}^{\prime}-\chi_{2}\left(n^{\prime}\right)\right]+\left[g_{2}^{\prime}-\chi_{2}\left(n^{\prime}\right), \chi_{2}(n)\right]\right) \\
& \quad-\left(\left[g_{2}, \chi_{2}\left(n^{\prime}\right)\right]+\left[\chi_{2}\left(n^{\prime}\right), g_{2}^{\prime}\right]\right) \\
= & y .
\end{aligned}
$$

b) follows directly from the proof of $a$ ).

Proposition 3.11 Let $\beta: \mathfrak{g} \rightarrow \mathfrak{h}$ be a homomorphism of Leibniz algebras. Then $\beta$ induces a Lie-isoclinic homomorphism from $\left(e_{\mathfrak{g}}\right)$ to $\left(e_{\mathfrak{h}}\right)$ if and only if $\operatorname{Ker}(\beta) \cap$ $[\mathfrak{g}, \mathfrak{g}]_{\text {Lie }}=0$ and $\operatorname{Im}(\beta)+Z_{\text {Lie }}(\mathfrak{h})=\mathfrak{h}$.

In this case we call $\beta$ a Lie-isoclinic homomorphism.

Proof. Assume that $\operatorname{Ker}(\beta) \cap[\mathfrak{g}, \mathfrak{g}]_{\text {Lie }}=0$ and $\operatorname{Im}(\beta)+Z_{\text {Lie }}(\mathfrak{h})=\mathfrak{h}$. First we prove that $\beta\left(Z_{\text {Lie }}(\mathfrak{g})\right) \subseteq Z_{\text {Lie }}(\mathfrak{h})$. Indeed let $h \in \mathfrak{h}$. Since $\operatorname{Im}(\beta)+Z_{\text {Lie }}(\mathfrak{h})=\mathfrak{h}$, it follows 
that $h=\beta(x)+h_{0}$ for some $x \in \mathfrak{g}$ and $h_{0} \in Z_{\text {Lie }}(\mathfrak{h})$. Then

$$
\begin{aligned}
{[\beta(g), h]+[h, \beta(g)] } & =\left[\beta(g), \beta(x)+h_{0}\right]+\left[\beta(x)+h_{0}, \beta(g)\right] \\
& =[\beta(g), \beta(x)]+[\beta(x), \beta(g)] \\
& =0 .
\end{aligned}
$$

So the maps $\alpha:=\left.\beta\right|_{Z_{\text {Lie }}(\mathfrak{g})}$ and $\gamma: \mathfrak{g} / Z_{\text {Lie }}(\mathfrak{g}) \rightarrow \mathfrak{h} / Z_{\text {Lie }}(\mathfrak{h})$ given by $\gamma(\bar{g})=\overline{\beta(g)}$ are well-defined homomorphisms and it is readily verified that $(\alpha, \beta, \gamma):\left(e_{\mathfrak{g}}\right) \rightarrow\left(e_{\mathfrak{h}}\right)$ is a homomorphism of Lie-central extensions. To show that it is Lie-isoclinic, it is enough to show by Proposition 3.10 that $\gamma$ is an isomorphism. To show that $\gamma$ is one-to-one, let $g \in \mathfrak{g}$ such that $\gamma(\bar{g})=0$. Then $\beta(g) \in Z_{\text {Lie }}(\mathfrak{h})$. We claim that $g \in Z_{\text {Lie }}(\mathfrak{g})$. Indeed if $g \notin Z_{\text {Lie }}(\mathfrak{g})$, then $m:=\left[g, g^{\prime}\right]+\left[g^{\prime}, g\right] \neq 0$ for some $g^{\prime} \in \mathfrak{g}$. But $\beta(m)=\left[\beta(g), \beta\left(g^{\prime}\right)\right]+\left[\beta\left(g^{\prime}\right), \beta(g)\right]=0$ because $\beta(g) \in Z_{\text {Lie }}(\mathfrak{h})$. This implies that $m \in \operatorname{Ker}(\beta) \cap[\mathfrak{g}, \mathfrak{g}]_{\text {Lie }}$, and thus $m=0$. A contradiction. Next we show that $\gamma$ is onto. Let $h \in \mathfrak{h}$. Since $\operatorname{Im}(\beta)+Z_{\text {Lie }}(\mathfrak{h})=\mathfrak{h}$, it follows that $h=\beta(x)+h_{0}$ for some $x \in \mathfrak{g}$ and $h_{0} \in Z_{\text {Lie }}(\mathfrak{h})$. Clearly, $\bar{h}=\overline{\beta(x)}=\gamma(\bar{x})$.

Conversely, assume that $\beta$ induces a Lie-isoclinic homomorphism $(\alpha, \beta, \gamma)$ from $\left(e_{\mathfrak{g}}\right)$ to $\left(e_{\mathfrak{h}}\right)$. Then again by Proposition $\left[3.10, \operatorname{Ker}(\beta) \cap[\mathfrak{g}, \mathfrak{g}]_{\text {Lie }}=0\right.$. It remains to show that $\operatorname{Im}(\beta)+Z_{\text {Lie }}(\mathfrak{h})=\mathfrak{h}$. Clearly $\operatorname{Im}(\beta)+Z_{\text {Lie }}(\mathfrak{h}) \subseteq \mathfrak{h}$. Now let $h \in \mathfrak{h}$. Following the notation in equation (3), $p r_{\mathfrak{h}}$ and $\gamma$ are onto, then $p r_{\mathfrak{h}}(h)=\gamma \circ p r_{\mathfrak{g}}(g)$ for some $g \in \mathfrak{g}$. On the other hand we have by the homomorphism $(\alpha, \beta, \gamma)$ that $\left(\gamma \circ p r_{\mathfrak{g}}\right)(g)=\left(p r_{\mathfrak{h}} \circ \beta\right)(h)$, which implies that $h-\beta(g) \in \operatorname{Ker}\left(p r_{\mathfrak{h}}\right)=Z_{\text {Lie }}(\mathfrak{h})$. Therefore $h=\beta(g)+n$ for some $n \in Z_{\text {Lie }}(\mathfrak{h})$. This completes the proof.

Lemma 3.12 Let $P=\{(a, c) \in A \times C: \alpha(a)=\beta(c)\}$ be the pull-back of $a$ homomorphism $\alpha: A \rightarrow B$ and an isomorphism $\beta: C \rightarrow B$ in the category of Leibniz algebras.

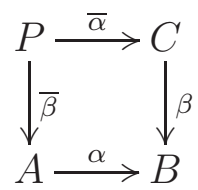

Then

a) $\beta$ monomorphism implies $\bar{\beta}$ monomorphism.

b) Given $\pi: G \rightarrow C, \gamma: G \rightarrow A$ such that $\alpha \circ \gamma=\beta \circ \pi$, then there exists a unique homomorphism $\omega: G \rightarrow P$ satisfying $\bar{\alpha} \circ \omega=\pi$ and $\bar{\beta} \circ \omega=\gamma$. If $\pi$ is an epimorphism, then $\omega$ is an epimorphism.

Proof. Direct checking.

\section{Proposition 3.13}

a) A homomorphism $(\alpha, \beta, \gamma):\left(g_{1}\right) \rightarrow\left(g_{2}\right)$ is Lie-isoclinic if and only if $\gamma$ is an isomorphism and $\beta: \mathfrak{g}_{1} \rightarrow \mathfrak{g}_{2}$ is a Lie-isoclinic homomorphism of Leibniz algebras. 
b) The composition of Lie-isoclinic homomorphisms is an isoclinic homomorphism.

c) Each Lie-isoclinic homomorphism is a composition of a Lie-isoclinic epimorphism and a Lie-isoclinic monomorphism.

Proof. a) Assume that $(\alpha, \beta, \gamma):\left(g_{1}\right) \rightarrow\left(g_{2}\right)$ is Lie-isoclinic. Then by Proposition $3.10, \gamma$ is an isomorphism and $\operatorname{Ker}(\beta) \cap\left[\mathfrak{g}_{1}, \mathfrak{g}_{1}\right]_{\text {Lie }}=0$. It remains to show that $\operatorname{Im}(\beta)+Z_{\text {Lie }}\left(\mathfrak{g}_{2}\right)=\mathfrak{g}_{2}$. Clearly $\operatorname{Im}(\beta)+Z_{\text {Lie }}\left(\mathfrak{g}_{2}\right) \subseteq \mathfrak{g}_{2}$. Now let $h \in \mathfrak{g}_{2}$. Since $\pi_{2}$ and $\gamma$ are onto, it follows that $\pi_{2}(h)=\gamma \circ \pi_{1}(g)$ for some $g \in \mathfrak{g}_{1}$. Also by the homomorphism $(\alpha, \beta, \gamma)$, we have $\left(\gamma \circ \pi_{1}\right)(g)=\left(\pi_{2} \circ \beta\right)(g)$. So $h-\beta(g) \in$ $\operatorname{Ker}\left(\pi_{2}\right)=\operatorname{Im}\left(\chi_{2}\right)$. Therefore $h=\beta(g)+\chi_{2}(n)$ for some $n \in \mathfrak{n}_{2}$. This proves the assertion since $\chi_{2}(n) \in Z_{\text {Lie }}\left(\mathfrak{g}_{2}\right)$ as $\left[\mathfrak{n}_{2}, \mathfrak{g}_{2}\right]_{\text {Lie }}=0$.

Conversely, assume that $\beta$ is a Lie-isoclinic homomorphism of Leibniz algebras. Then $\operatorname{Ker}(\beta) \cap\left[\mathfrak{g}_{1}, \mathfrak{g}_{1}\right]_{\text {Lie }}=0$ and $\operatorname{Im}(\beta)+Z_{\text {Lie }}\left(\mathfrak{g}_{2}\right)=\mathfrak{g}_{2}$. Again by Proposition 3.10, it is enough to show that $\gamma$ is an isomorphism. To show that $\gamma$ is onto, let $q \in \mathfrak{q}_{2}$ and let $h \in \mathfrak{g}_{2}$ such that $q=\pi_{2}(h)$. Then $h=\beta(x)+h_{0}$ for some $x \in \mathfrak{g}_{1}$ and $h_{0} \in Z_{\text {Lie }}\left(\mathfrak{g}_{2}\right)$. We then have $\gamma\left(\pi_{1}(x)\right)=\pi_{2}(\beta(x))=\pi_{2}\left(\beta(x)+h_{0}\right)=\pi_{2}(h)=q$. To show that $\gamma$ is one-to-one, let $q_{1} \in \mathfrak{q}_{1}$ with $\gamma\left(q_{1}\right)=0$, i.e. $\gamma\left(\pi_{1}\left(g_{1}\right)\right)=0$ for some $g_{1} \in \mathfrak{g}_{1}$. By the homomorphism $(\alpha, \beta, \gamma)$, it follows that $\pi_{2}\left(\beta\left(g_{1}\right)\right)=0$ i.e. $\beta\left(g_{1}\right)=\chi_{2}(n)$ for some $n \in \mathfrak{n}_{2}$. We claim that $g_{1} \in \mathfrak{n}_{1}$ in which case $q_{1}=\pi_{1}\left(g_{1}\right)=$ 0 . Otherwise, there exists $g_{1}^{\prime} \in \mathfrak{g}_{1}$ such that $m:=\left[g_{1}, g_{1}^{\prime}\right]+\left[g_{1}^{\prime}, g_{1}\right] \neq 0$. So

$$
\begin{aligned}
\beta(m) & =\left[\beta\left(g_{1}\right), \beta\left(g_{1}^{\prime}\right)\right]+\left[\beta\left(g_{1}^{\prime}\right), \beta\left(g_{1}\right)\right] \\
& =\left[\chi_{2}(n), \beta\left(g_{1}^{\prime}\right)\right]+\left[\beta\left(g_{1}^{\prime}\right), \chi_{2}(n)\right] \\
& =0 .
\end{aligned}
$$

This contradicts the fact that $\operatorname{Ker}(\beta) \cap\left[\mathfrak{g}_{1}, \mathfrak{g}_{1}\right]_{\text {Lie }}=0$.

The proof of $b$ ) is straightforward.

c) Let $\beta: \mathfrak{g} \rightarrow \mathfrak{h}$ be a Lie-isoclinic homomorphism. Then $\beta$ induces a Lieisoclinism $(\alpha, \beta, \gamma):\left(e_{\mathfrak{g}}\right) \rightarrow\left(e_{\mathfrak{h}}\right)$ where $\alpha=\beta_{\mid Z_{\text {Lie }}(\mathfrak{g})}, \gamma=\bar{\beta}$, that is $\gamma(\bar{g})=$ $\overline{\beta(g)}$. Moreover, by Proposition 3.10, we know that $\gamma$ is an isomorphism and $\operatorname{Ker}(\beta) \cap[\mathfrak{g}, \mathfrak{g}]_{\text {Lie }}=0$. Equivalently, by Definition 3.9, $\beta^{\prime}:[\mathfrak{g}, \mathfrak{g}]_{\text {Lie }} \rightarrow[\mathfrak{h}, \mathfrak{h}]_{\text {Lie }}$ is an isomorphism and $\left(\gamma, \beta^{\prime}\right):\left(e_{\mathfrak{g}}\right) \sim\left(e_{\mathfrak{h}}\right)$. Now applying Proposition 3.7 we obtain the decomposition

$$
\left(e_{\mathfrak{g}}\right) \stackrel{\left(\bar{\omega}_{\bar{\omega}}, \omega, 1\right)}{\rightarrow}\left(e_{\mathfrak{h}} \gamma\right) \stackrel{(1, \bar{\gamma}, \gamma)}{\rightarrow}\left(e_{\mathfrak{h}}\right)
$$

where $w$ is induced by pull-back properties. 
In fact we have the following diagram:



Now Lemma 3.12 implies that $\omega$ is an epimorphism and $\bar{\gamma}$ is a monomorphism, as required.

In the following consider an isomorphism $\eta: \mathfrak{q}_{1} \rightarrow \mathfrak{q}_{2}$ and put $\mathfrak{q}=\mathfrak{q}_{1}$. Construct the following backward (see Proposition [3.7) and product Lie-central extensions:

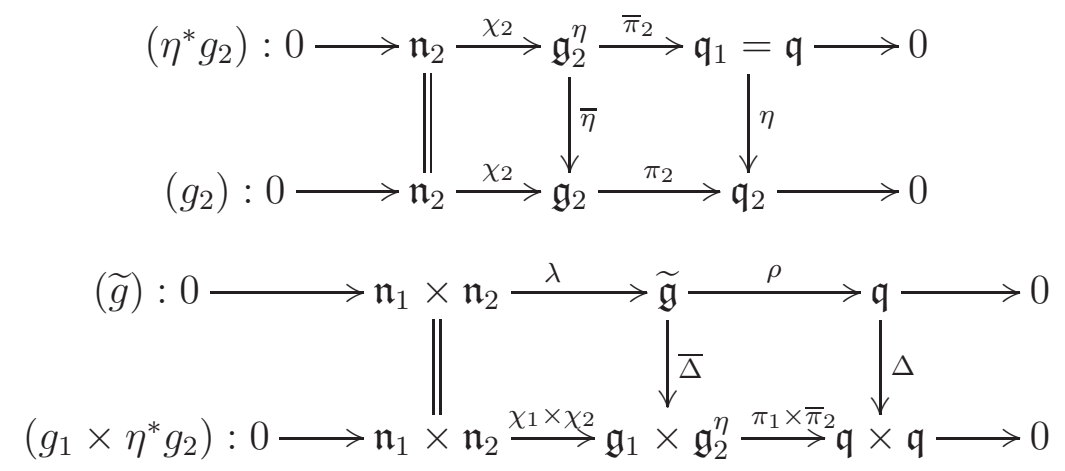

where $\Delta(q)=(q, q), q \in \mathfrak{q}$ is the diagonal map and $\widetilde{\mathfrak{g}}=\left(\mathfrak{g}_{1} \times \mathfrak{g}_{2}^{\eta}\right)^{\Delta}$.

An easy computation shows that $\widetilde{\mathfrak{g}} \equiv\left\{\left(g_{1}, g_{2}\right), g_{i} \in \mathfrak{g}_{i}, i=1,2 \mid \eta \circ \pi_{1}\left(g_{1}\right)=\right.$ $\left.\pi_{2}\left(g_{2}\right)\right\}, \rho\left(g_{1}, g_{2}\right)=\pi_{1}\left(g_{1}\right), \lambda \equiv \chi_{1} \times \chi_{2}$ and $(\widetilde{g})$ is a Lie-central extension.

Furthermore we denote by $\sigma_{i}: \mathfrak{n}_{1} \times \mathfrak{n}_{2} \rightarrow \mathfrak{n}_{i}$ and $\tau_{i}: \widetilde{\mathfrak{g}} \rightarrow \mathfrak{g}_{i}$ the $i$-th projection $(i=1,2)$ and put $\gamma_{1}=i d_{\mathfrak{q}}: \mathfrak{q} \rightarrow \mathfrak{q}_{1}, \gamma_{2}=\eta: \mathfrak{q} \rightarrow \mathfrak{q}_{2}$.

\section{Proposition 3.14}

a) The following diagrams are commutative for $i=1,2$ :

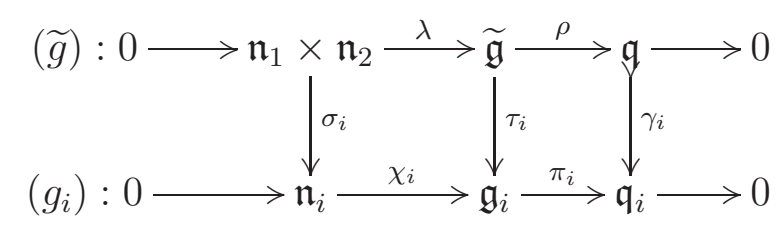

b) The isomorphism $\eta: \mathfrak{q}_{1} \rightarrow \mathfrak{q}_{2}$ induces a Lie-isoclinism from $\left(g_{1}\right)$ to ( $\left.g_{2}\right)$ if and only if $\left(\sigma_{i}, \tau_{i}, \gamma_{i}\right), i=1,2$, are Lie-isoclinic epimorphisms. 
Proof. a) Let $\left(g_{1}, g_{2}\right) \in \widetilde{\mathfrak{g}}$. Then for $i=1,2$, we have

$$
\left(\gamma_{i} \circ \rho\right)\left(g_{1}, g_{2}\right)=\gamma_{i}\left(\pi_{1}\left(g_{1}\right)\right)=\left\{\begin{array}{ll}
\pi_{1}\left(g_{1}\right), & \text { if } i=1 \\
\eta\left(\pi_{1}\left(g_{1}\right)\right), & \text { if } i=2
\end{array}=\left(\pi_{i} \circ \tau_{i}\right)\left(g_{1}, g_{2}\right) .\right.
$$

On the other hand, let $\left(n_{1}, n_{2}\right) \in \mathfrak{n}_{1} \times \mathfrak{n}_{2}$. Then for $i=1,2$,

$$
\left(\chi_{i} \circ \sigma_{i}\right)\left(n_{1}, n_{2}\right)=\chi_{i}\left(n_{i}\right)=\tau_{i}\left(\chi\left(n_{1}\right), \chi\left(n_{2}\right)\right)=\left(\tau_{i} \circ \lambda\right)\left(n_{1}, n_{2}\right) .
$$

b) Assume that $\left(\sigma_{i}, \tau_{i}, \gamma_{i}\right), i=1,2$ are Lie-isoclinic epimorphisms. Then by Proposition 3.10, $\operatorname{Ker}\left(\tau_{i}\right) \cap[\widetilde{\mathfrak{g}}, \widetilde{\mathfrak{g}}]_{\text {Lie }}=0$, and $\gamma_{i}$ are isomorphisms, $i=1,2$.

Define $\eta:=\gamma_{2}$ and $\xi:\left[\mathfrak{g}_{1}, \mathfrak{g}_{1}\right]_{\text {Lie }} \rightarrow\left[\mathfrak{g}_{2}, \mathfrak{g}_{2}\right]_{\text {Lie }}$ by $\xi(g)=h$ where $\pi_{2}(h)=$ $\eta \circ \pi_{1}(g) . \xi$ is a well-defined homomorphism and the diagram

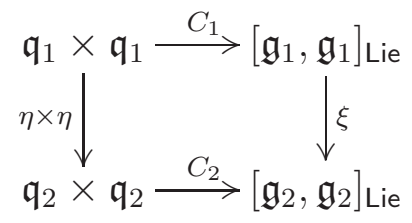

is commutative. It remains to show that $\xi$ is bijective.

$\xi$ is one-to-one, since for any $g \in\left[\mathfrak{g}_{1}, \mathfrak{g}_{1}\right]_{\text {Lie }}$ such that $\xi(g)=0$, we have that $(g, \xi(g)) \in \operatorname{Ker}\left(\tau_{2}\right) \cap[\widetilde{\mathfrak{g}}, \widetilde{\mathfrak{g}}]_{\text {Lie }}=0$, which implies that $g=0$.

$\xi$ is onto, since for any $h=\left[b_{1}, b_{2}\right]+\left[b_{2}, b_{1}\right] \in\left[\mathfrak{g}_{2}, \mathfrak{g}_{2}\right]_{\text {Lie }}$ we have that there exists $a_{j} \in \mathfrak{g}_{1}, j=1,2$ with $\pi_{2}\left(b_{j}\right)=\eta \circ \pi_{1}\left(a_{j}\right)$ such that $\tau_{2}\left(a_{j}, b_{j}\right)=b_{j}$. Let be $g:=\left[a_{1}, a_{2}\right]+\left[a_{2}, a_{1}\right]$. Clearly, $\pi_{2}(h)=\eta \circ \pi_{1}(g)$. So $\pi_{2}(\xi(g))=\pi_{2}(h)$ by definition of $\xi$. Therefore $h=\xi(g)+\chi_{2}(n)$ for some $n \in \mathfrak{n}_{2}$. However, it is obvious that $\left(0, \chi_{2}(n)\right) \in \operatorname{Ker}\left(\tau_{1}\right) \cap[\widetilde{\mathfrak{g}}, \widetilde{\mathfrak{g}}]_{\text {Lie }}=0$. This implies that $\chi_{2}(n)=0$, and thus $h=\xi(g)$.

Conversely, Suppose that $\eta: \mathfrak{q}_{1} \rightarrow \mathfrak{q}_{2}$ induces a Lie-isoclinism $(\eta, \xi):\left(g_{1}\right) \rightarrow$ $\left(g_{2}\right)$. Then we have an isomorphism $\xi:\left[\mathfrak{g}_{1}, \mathfrak{g}_{1}\right]_{\text {Lie }} \rightarrow\left[\mathfrak{g}_{2}, \mathfrak{g}_{2}\right]_{\text {Lie }}$ defined by $\xi\left(\left[g_{1}, g_{2}\right]+\right.$ $\left.\left[g_{2}, g_{1}\right]\right)=\left[h_{1}, h_{2}\right]+\left[h_{2}, h_{1}\right], g_{i} \in \mathfrak{g}_{1}, h_{i} \in \mathfrak{g}_{2}, \eta \circ \pi_{1}\left(g_{i}\right)=\pi_{2}\left(h_{i}\right), i=1,2$. Thus $\left(g_{i}, h_{i}\right) \in \widetilde{\mathfrak{g}}, i=1,2$, and we obtain

$$
\begin{aligned}
& {\left[\left(g_{1}, h_{1}\right),\left(g_{2}, h_{2}\right)\right]+\left[\left(g_{2}, h_{2}\right),\left(g_{1}, h_{1}\right)\right]=} \\
& \left(\left[g_{1}, g_{2}\right]+\left[g_{2}, g_{1}\right],\left[h_{1}, h_{2}\right]+\left[h_{2}, h_{1}\right]\right)= \\
& \left(\left[g_{1}, g_{2}\right]+\left[g_{2}, g_{1}\right], \xi\left(\left[g_{1}, g_{2}\right]+\left[g_{2}, g_{1}\right]\right)\right) .
\end{aligned}
$$

This implies that $[\widetilde{\mathfrak{g}}, \widetilde{\mathfrak{g}}]_{\text {Lie }}=\left\{\left(g^{\prime}, \xi\left(g^{\prime}\right) \mid g^{\prime} \in\left[\mathfrak{g}_{1}, \mathfrak{g}_{1}\right]_{\text {Lie }}\right\}\right.$.

For the case of $i=1, \gamma_{1}=I d_{\mathfrak{q}_{1}}$. Now let $\left(g^{\prime}, \xi\left(g^{\prime}\right)\right) \in \operatorname{Ker}\left(\tau_{1}\right) \cap[\widetilde{\mathfrak{g}}, \widetilde{\mathfrak{g}}]_{\text {Lie }}$. Then $\tau_{1}\left(g^{\prime}, \xi\left(g^{\prime}\right)\right)=g^{\prime}=0$, hence $\xi\left(g^{\prime}\right)=0$ and thus $\operatorname{Ker}\left(\tau_{1}\right) \cap[\widetilde{\mathfrak{g}}, \mathfrak{\mathfrak { g }}]_{\text {Lie }}=0$. Proposition 3.10 implies that $\left(g_{1}\right) \sim(\widetilde{g})$.

For the case of $i=2, \gamma_{2}=\eta$ and the fact $\operatorname{Ker}\left(\tau_{2}\right) \cap[\widetilde{\mathfrak{g}}, \widetilde{\mathfrak{g}}]_{\text {Lie }}=0$ can be proved in an analogous way as case $i=1$. Proposition 3.10 implies that $\left(g_{2}\right) \sim(\widetilde{g})$. 
Let $(g): 0 \rightarrow \mathfrak{n} \stackrel{\chi}{\rightarrow} \mathfrak{g} \stackrel{\pi}{\rightarrow} \mathfrak{q} \rightarrow 0$ be a Lie-central extension and $\mathfrak{a}:=\mathfrak{h} /[\mathfrak{h}, \mathfrak{h}]_{\text {Lie }}$ where $\mathfrak{h}$ is a Leibniz algebra. Let $\varphi: \mathfrak{g} \times \mathfrak{a} \rightarrow \mathfrak{g}$ be the projection onto $\mathfrak{g}$, then

$$
(g \times a): 0 \rightarrow \mathfrak{n} \times \mathfrak{a} \stackrel{\chi \times i d}{\rightarrow} \mathfrak{g} \times \mathfrak{a} \stackrel{\pi \circ \varphi}{\rightarrow} \mathfrak{q} \rightarrow 0
$$

is a Lie-central extension.

Let $\mu: \mathfrak{g} \rightarrow \mathfrak{g} \times \mathfrak{a}, \mu(g)=(g, 0) ; \varphi^{\prime}: \mathfrak{n} \times \mathfrak{a} \rightarrow \mathfrak{n}, \varphi^{\prime}(n, a)=n ; \mu^{\prime}: \mathfrak{n} \rightarrow$ $\mathfrak{n} \times \mathfrak{a}, \mu^{\prime}(n)=(n, 0)$ be. Then the diagrams:

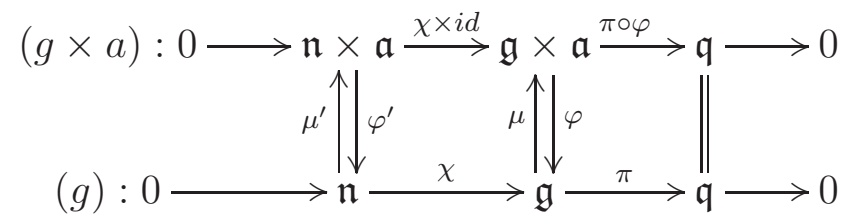

are commutative, $\left(\varphi^{\prime}, \varphi, i d_{\mathfrak{q}}\right):(g \times a) \rightarrow(g)$ is a Lie-isoclinic epimorphism and $\left(\mu^{\prime}, \mu, i d_{\mathfrak{q}}\right):(g) \rightarrow(g \times a)$ is a Lie-isoclinic monomorphism.

Let $\left(g_{i}\right), \sigma_{i}, \tau_{i}, \gamma_{i}, i=1,2$, and $(\widetilde{g})$ be as in (4), $\eta: \mathfrak{q}_{1} \rightarrow \mathfrak{q}_{2}$ an isomorphism and $\mathfrak{a}=\widetilde{\mathfrak{g}} /[\widetilde{\mathfrak{g}}, \widetilde{\mathfrak{g}}]_{\text {Lie }}$, and $\alpha: \mathfrak{n}_{1} \times \mathfrak{n}_{2} \rightarrow \mathfrak{n}_{1} \times \mathfrak{a}, \alpha(n)=\left(\sigma_{1}(n), \overline{\lambda(n)}\right) ; \beta: \widetilde{\mathfrak{g}} \rightarrow$ $\mathfrak{g}_{1} \times \mathfrak{a}, \beta(g)=\left(\tau_{1}(g), \bar{g}\right)$.

Lemma 3.15 Assume that $\eta$ induces a Lie-isoclinism from $\left(g_{1}\right)$ to $\left(g_{2}\right)$. Then $\left(\alpha, \beta, \gamma_{1}\right):(\widetilde{g}) \rightarrow\left(g_{1} \times a\right)$ is a Lie-isoclinic monomorphism.

Proof. Let $g \in \operatorname{Ker}(\beta) \cap[\widetilde{\mathfrak{g}}, \widetilde{\mathfrak{g}}]_{\text {Lie }}$. Then $\beta(g)=0$ i.e. $\left(\tau_{1}(g), \bar{g}\right)=0$ which implies that $\tau_{1}(g)=0$ and $\bar{g}=0$. So $g \in \operatorname{Ker}\left(\tau_{1}\right) \cap[\widetilde{\mathfrak{g}}, \mathfrak{\mathfrak { g }}]_{\text {Lie }}=0$ by Proposition 3.14. Hence $g=0$ and thus $\operatorname{Ker}(\beta) \cap[\widetilde{\mathfrak{g}}, \mathfrak{\mathfrak { g }}]_{\text {Lie }}=0$. To show that $\beta$ is one-to-one, notice that $\bar{g}=0$ iff $g \in[\widetilde{\mathfrak{g}}, \widetilde{\mathfrak{g}}]_{\text {Lie }}$. So $\beta(g)=0$ iff $g \in \operatorname{Ker}\left(\tau_{1}\right) \cap[\widetilde{\mathfrak{g}}, \widetilde{\mathfrak{g}}]_{\text {Lie }}=0$, and thus $g=0$.

Let $(\eta, \xi):\left(g_{1}\right) \sim\left(g_{2}\right)$ be a Lie-isoclinism. Now we consider the following commutative diagram where $\mathfrak{a}:=\widetilde{\mathfrak{g}} /[\widetilde{\mathfrak{g}}, \widetilde{\mathfrak{g}}]_{\text {Lie }}$ : 


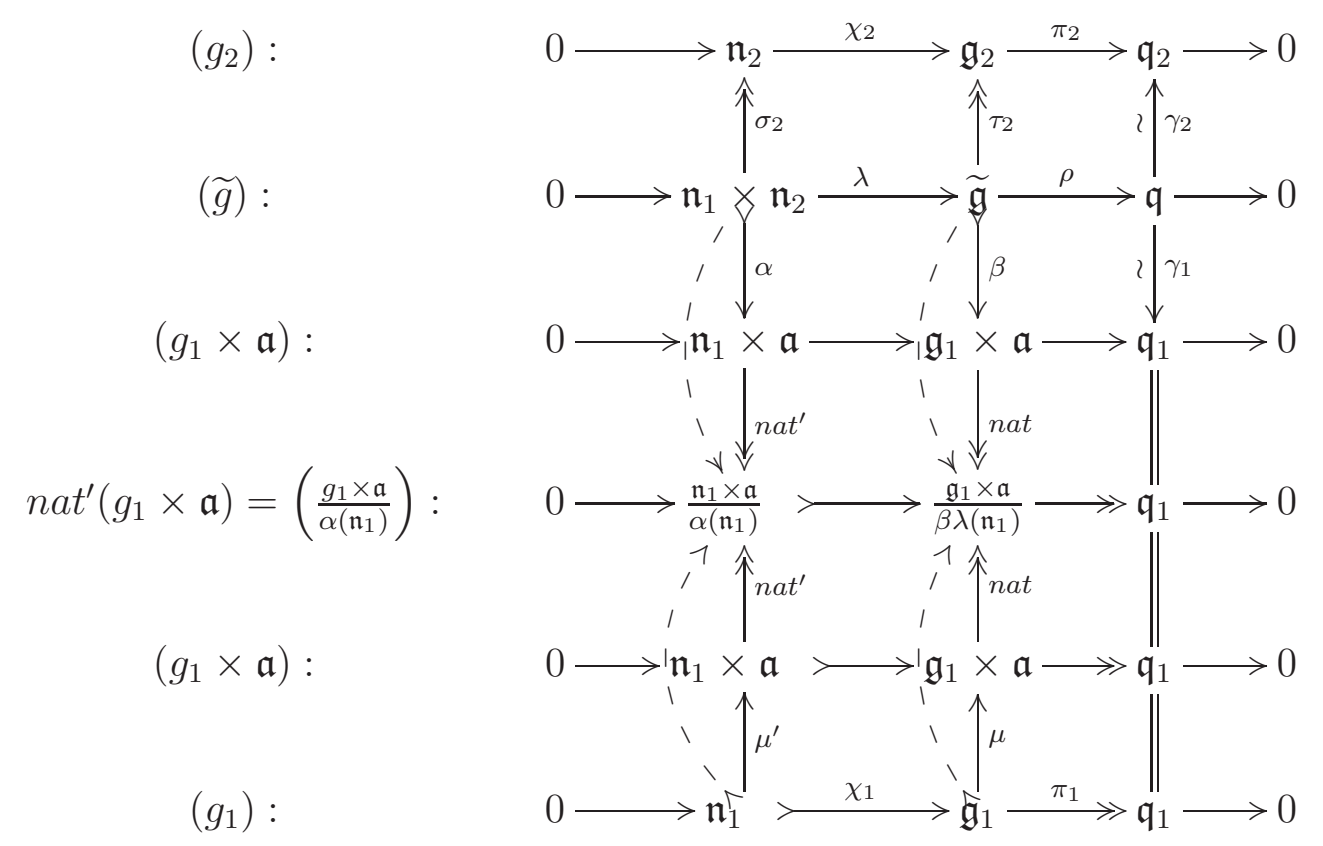

The following holds:

$$
\begin{gathered}
\alpha\left(\mathfrak{n}_{1}\right)=\left\{\left(n_{1},\left(\overline{\left.\chi_{1}\left(n_{1}\right), 0\right)}\right), \text { for all } n_{1} \in \mathfrak{n}_{1}\right\}\right. \\
\operatorname{Ker}(n a t)=\left\{\left(\chi_{1}\left(n_{1}\right), \overline{\left(\chi_{1}\left(n_{1}\right), 0\right)}\right), \text { for all } n_{1} \in \mathfrak{n}_{1}\right\} \\
{[\widetilde{\mathfrak{g}}, \widetilde{\mathfrak{g}}]_{\text {Lie }}=\left\{[g, \xi(g)], \text { for all } g \in\left[\mathfrak{g}_{1}, \mathfrak{g}_{1}\right]_{\text {Lie }}\right\} .}
\end{gathered}
$$

This yields:

$$
\begin{gathered}
\operatorname{Ker}(\text { nat }) \cap\left[\mathfrak{g}_{1} \times \mathfrak{a}, \mathfrak{g}_{1} \times \mathfrak{a}\right]_{\text {Lie }}=0 \\
\alpha\left(\mathfrak{n}_{1}\right) \cap \mu^{\prime}\left(\mathfrak{n}_{1}\right)=0 .
\end{gathered}
$$

Furthermore we have

$$
\operatorname{Ker}\left(n a t^{\prime} \circ \alpha\right)=\mathfrak{n}_{1}=\operatorname{Ker}\left(\sigma_{2}\right)
$$

and we obtain:

\section{Lemma 3.16}

a) The composition of $\left(\right.$ nat $^{\prime}$, nat, $\left.I d_{\mathfrak{q}_{1}}\right)$ and $\left(\mu^{\prime}, \mu, I d_{\mathfrak{q}_{1}}\right)$ is a Lie-isoclinic monomorphism from $\left(g_{1}\right)$ into nat $^{\prime}\left(\left(g_{1} \times \mathfrak{a}\right)\right)$.

b) The composition of $\left(\right.$ nat $^{\prime}$, nat, $\left.I d_{\mathfrak{q}_{1}}\right)$ and $\left(\alpha, \beta, \gamma_{1}\right)$ induces a Lie-isoclinic monomorphism from $\left(g_{2}\right)$ into nat $^{\prime}\left(\left(g_{1} \times \mathfrak{a}\right)\right)$. 
Proof. a) Let $g \in \operatorname{Ker}($ nat $\circ \mu) \cap\left[\mathfrak{g}_{1}, \mathfrak{g}_{1}\right]_{\text {Lie }}$. Then $\mu(g) \in \operatorname{Ker}($ nat $)$ and $g=$ $\left[g_{1}, g_{2}\right]+\left[g_{2}, g_{1}\right]$ for some $g_{1}, g_{2} \in \mathfrak{g}_{1}$. So $\mu(g)=\left[\mu\left(g_{1}\right), \mu\left(g_{2}\right)\right]+\left[\mu\left(g_{2}\right), \mu\left(g_{1}\right)\right] \in$ $\left[\mathfrak{g}_{1} \times \mathfrak{a}, \mathfrak{g}_{1} \times \mathfrak{a}\right]_{\text {Lie }}$. Thus $(g, 0)=\mu(g) \in \operatorname{Ker}(n a t) \cap\left[\mathfrak{g}_{1} \times \mathfrak{a}, \mathfrak{g}_{1} \times \mathfrak{a}\right]_{\text {Lie }}=0$ by (10). Hence the composition $\left(\right.$ nat $^{\prime}$, nat, $\left.I d_{\mathfrak{q}_{1}}\right)$ and $\left(\mu^{\prime}, \mu, I d_{\mathfrak{q}_{1}}\right)$ is a Lie-isoclinism by Proposition 3.10 .

To show that nat $\circ \mu$ is one-to-one, let $g \in \mathfrak{g}_{1}$ such that $($ nat $\circ \mu)(g)=0$. This implies that $(g, 0) \in \beta \lambda\left(\mathfrak{n}_{1}\right)=\left(\chi_{1}(n), \overline{\left(\chi_{1}(n), 0\right)}\right)$ for some $n \in \mathfrak{n}_{1}$. So $g=\chi_{1}(n)$ and $\left(\chi_{1}(n), 0\right) \in[\widetilde{\mathfrak{g}}, \widetilde{\mathfrak{g}}]_{\text {Lie. }}$. Therefore $(g, 0) \in \operatorname{Ker}\left(\tau_{2}\right) \cap[\widetilde{\mathfrak{g}}, \widetilde{\mathfrak{g}}]_{\text {Lie }}$. Since $(\eta, \xi)$ is a Lie-isoclinism from $\left(g_{1}\right)$ to $\left(g_{2}\right)$, it follows by the proof of Proposition 3.14 that $\operatorname{Ker}\left(\tau_{2}\right) \cap[\widetilde{\mathfrak{g}}, \widetilde{\mathfrak{g}}]_{\text {Lie }}=0$. Hence $g=0$.

b) Consider the map $\delta: \mathfrak{g}_{2} \rightarrow \frac{\mathfrak{g}_{1} \times \mathfrak{a}}{\beta \lambda\left(\mathfrak{n}_{1}\right)}$ defined by $\delta(h)=($ nat $\circ \beta)(x, h)$ where $\pi_{2}(h)=\eta\left(\pi_{1}(x)\right)$ for some $x \in \mathfrak{g}_{1}$.

$\delta$ is well defined, since for $x, x^{\prime} \in \mathfrak{g}_{1}$ such that $\eta\left(\pi_{1}(x)\right)=\pi_{2}(h)=\eta\left(\pi_{1}\left(x^{\prime}\right)\right)$, then $x-x^{\prime} \in \operatorname{Ker}\left(\pi_{1}\right)$, that is $x-x^{\prime}=\chi_{1}(n)$ for some $n \in \mathfrak{g}_{1}$. We then have

$$
(x, \overline{(x, h)})-\left(x^{\prime}, \overline{\left(x^{\prime}, h\right)}\right)=\left(x-x^{\prime}, \overline{\left(x-x^{\prime}, 0\right)}\right)=\left(\chi_{1}(n), \overline{\left(\chi_{1}(n), 0\right)}\right) \in \beta \lambda\left(\mathfrak{n}_{1}\right) .
$$

Hence $($ nat $\circ \beta)(x, h)=($ nat $\circ \beta)\left(x^{\prime}, h\right)$.

Let $\delta_{0}$ be the restriction of $\delta$ to $\mathfrak{n}_{2}$. It is easy to check that $\left(\delta_{0}, \delta, \eta^{-1}\right):\left(g_{2}\right) \rightarrow$ $n a t^{\prime}\left(g_{1} \times \mathfrak{a}\right)$ is a homomorphism of Lie-central extensions.

Let $h \in \operatorname{Ker}(\delta)$. Then $(x, \overline{(x, h)}) \in \beta \lambda\left(\mathfrak{n}_{1}\right)$ for some $x \in \mathfrak{g}_{1}$ satisfying $\pi_{2}(h)=$ $\eta\left(\pi_{1}(x)\right)$. Then $(x, \overline{(x, h)})=\left(\chi_{1}(n), \overline{\left(\chi_{1}(n), 0\right)}\right)$ for some $n \in \mathfrak{n}_{1}$. So $x=\chi_{1}(n)$ and $(0, h)=\left(x-\chi_{1}(n), h\right) \in[\widetilde{\mathfrak{g}}, \widetilde{\mathfrak{g}}]_{\text {Lie }}$. So $(0, h) \in \operatorname{Ker}\left(\tau_{1}\right) \cap[\widetilde{\mathfrak{g}}, \widetilde{\mathfrak{g}}]_{\text {Lie }}=0$ by the proof of Proposition 3.14. Therefore $h=0$. So $\delta$ is one to one. This implies that $\operatorname{Ker}(\delta) \cap\left[\mathfrak{g}_{2}, \mathfrak{g}_{2}\right]_{\text {Lie }}=0$. It follows by Proposition 3.10 that $\left(\delta_{0}, \delta, \eta^{-1}\right)$ is a Lieisoclinic monomorphism.

Theorem 3.17 The following statements are equivalent:

a) The Lie-central extensions $\left(g_{1}\right)$ and $\left(g_{2}\right)$ are Lie-isoclinic.

b) There exists a Lie-central extension $\left(g^{\prime}\right)$ together with Lie-isoclinic epimorphism from $\left(g^{\prime}\right)$ onto $\left(g_{1}\right)$ and $\left(g_{2}\right)$.

c) There exists a Lie-central extension $\left(g^{\prime \prime}\right)$ together with Lie-isoclinic monomorphisms from $\left(g_{1}\right)$ and $\left(g_{2}\right)$ into $\left(g^{\prime \prime}\right)$.

Proof. a) $\Longleftrightarrow$ b) Apply Proposition 3.14 b) by letting $\left(g^{\prime}\right)=(\tilde{g})$.

a) $\Longrightarrow$ c) Apply Lemma 3.16 by letting $\left(g^{\prime \prime}\right)=\left(\operatorname{nat}^{\prime}\left(g_{1} \times \mathfrak{a}\right)\right)$.

c) $\Longrightarrow$ a) Direct consequence of Proposition 3.5 .

Remark 3.18 If $\left(g_{1}\right)$ and $\left(g_{2}\right)$ are finite-dimensional, extensions $\left(g^{\prime}\right)$ and $\left(g^{\prime \prime}\right)$ in Theorem 3.17 can also be chosen finite-dimensional. The situation in Theorem 3.17 can be roughly sketched by $\left(g_{1}\right) \nleftarrow\left(g^{\prime}\right) \rightarrow\left(g_{2}\right)$ and $\left(g_{1}\right) \longmapsto\left(g^{\prime \prime}\right) \longleftrightarrow\left(g_{2}\right)$. In 
particular, the equivalence relations for Lie-central extensions generated by Lieisoclinic epimorphisms, respectively Lie-isoclinic monomorphisms, coincide with Lie-isoclinism.

Theorem 3.19 The following statements are equivalent:

a) $\left(g_{1}\right)$ and $\left(g_{2}\right)$ are Lie-isoclinic.

b) There exist a Leibniz algebra with trivial Lie-commutator $\mathfrak{a}$, a Lie-central extension $\left(g^{\prime}\right)$, a Lie-isoclinic monomorphism from $\left(g^{\prime}\right)$ into $\left(g_{1} \times \mathfrak{a}\right)$ and a Lie-isoclinic epimorphism from $\left(g^{\prime}\right)$ onto $\left(g_{2}\right)$.

c) There exist a Leibniz algebra with trivial Lie-commutator $\mathfrak{b}$, a Lie-central extension $\left(g^{\prime \prime}\right)$, a Lie-isoclinic epimorphism from $\left(g_{1} \times \mathfrak{b}\right)$ onto $\left(g^{\prime \prime}\right)$, and a Lie-isoclinic monomorphism from $\left(g_{2}\right)$ into $\left(g^{\prime \prime}\right)$.

Proof. a) $\Longrightarrow$ b) It follows from Proposition 3.14 and Lemmas 3.15 and 3.16 by letting $\mathfrak{a}=\tilde{\mathfrak{g}} /[\tilde{\mathfrak{g}}, \tilde{\mathfrak{g}}]_{\text {Lie }}$ and $\left(g^{\prime}\right)=(\tilde{\mathfrak{g}})$.

b) $\Longrightarrow$ c) By letting $\mathfrak{b}=\tilde{\mathfrak{g}} /[\tilde{\mathfrak{g}}, \tilde{\mathfrak{g}}]$ Lie and $\left(g^{\prime \prime}\right)=\left(\right.$ nat $\left.^{\prime}\left(g_{1} \times \mathfrak{a}\right)\right)$, the implication follows by definition of $\left(\operatorname{nat}^{\prime}\left(g_{1} \times \mathfrak{a}\right)\right)$ and the proof of Lemma 3.16 $\left.b\right)$.

c) $\Longrightarrow a$ ) It suffices to have in mind the following chain of Lie-isoclinisms:

$$
\left(g_{1}\right) \longleftrightarrow\left(g_{1} \times \mathfrak{b}\right) \longrightarrow\left(g^{\prime \prime}\right) \longleftrightarrow\left(g_{2}\right)
$$

Proposition 3.20 Let $\mathfrak{g}$ be a Leibniz algebra.

a) Let $\mathfrak{a}$ be a Leibniz algebra with trivial Lie-commutator. Then $\mathfrak{g}$ and $\mathfrak{g} \times \mathfrak{a}$ are Lie-isoclinic.

b) Let $\mathfrak{n}$ be a two-sided ideal of $\mathfrak{g}$. The natural homomorphism nat: $\mathfrak{g} \rightarrow \mathfrak{g} / \mathfrak{n}$ is a Lie-isoclinic epimorphism if and only if $\mathfrak{n} \cap[\mathfrak{g}, \mathfrak{g}]_{\text {Lie }}=0$.

c) Let $\mathfrak{h}$ be a subalgebra of $\mathfrak{g}$. The embedding of $\mathfrak{h}$ into $\mathfrak{g}$ is a Lie-isoclinic monomorphism if and only $\mathfrak{h}+Z_{\text {Lie }}(\mathfrak{g})=\mathfrak{g}$.

Proof. a) Notice that for a Leibniz algebra $\mathfrak{g}$ and a Leibniz algebra with trivial Lie-commutator $\mathfrak{a}$, we have $Z_{\text {Lie }}(\mathfrak{g} \times \mathfrak{a})=Z_{\text {Lie }}(\mathfrak{g}) \times \mathfrak{a}$.

The canonical inclusion $\beta: \mathfrak{g} \rightarrow \mathfrak{g} \times \mathfrak{a}, \beta(g)=(g, 0)$, satisfies that $\operatorname{Ker}(\beta) \cap$ $[\mathfrak{g}, \mathfrak{g}]_{\text {Lie }}=0$ and any element $(g, a) \in \mathfrak{g} \times \mathfrak{a}$ can be written as $(g, a)=(g, 0)+$ $(0, a) \in \operatorname{Im}(\beta)+Z_{\text {Lie }}(\mathfrak{g} \times \mathfrak{a})$. Now Proposition 3.11 ends the proof.

b) Follows by Proposition 3.11 since $\operatorname{Ker}($ nat $)=\mathfrak{n}$. In addition, if $\mathfrak{n} \cap[\mathfrak{g}, \mathfrak{g}]_{\text {Lie }} \neq$ 0 , then nat is not a Lie-isoclinic homomorphism by Proposition 3.10 .

Conversely, if $\mathfrak{n} \cap[\mathfrak{g}, \mathfrak{g}]_{\text {Lie }}=0$, then $\xi:[\mathfrak{g}, \mathfrak{g}]_{\text {Lie }} \rightarrow[\mathfrak{g} / \mathfrak{n}, \mathfrak{g} / \mathfrak{n}]_{\text {Lie }}, \xi\left(\left[g_{1}, g_{2}\right]+\right.$ $\left.\left[g_{2}, g_{1}\right]\right)=\left[g_{1}+\mathfrak{n}, g_{2}+\mathfrak{n}\right]+\left[g_{2}+\mathfrak{n}, g_{1}+\mathfrak{n}\right]$, is an isomorphism and $Z_{\text {Lie }}(\mathfrak{g} / \mathfrak{n})=\frac{Z_{\text {Lie }}(\mathfrak{g})}{\mathfrak{n}}$, 
and $\frac{\mathfrak{g}}{Z_{\text {Lie }}(\mathfrak{g})} \stackrel{\eta}{\cong} \frac{\frac{\mathfrak{g}}{\mathfrak{n}}}{\frac{Z_{\text {Lie }}(\mathfrak{g})}{\mathfrak{n}}}$ by the third isomorphism theorem. Now the commutativity of diagram (2) immediately follows.

c) Since $\operatorname{Im}(\mathfrak{h} \hookrightarrow \mathfrak{g})=\mathfrak{h}$ and $\operatorname{Ker}(\mathfrak{h} \hookrightarrow \mathfrak{g})=0$, then Proposition 3.11 ends the proof.

Theorem 3.21 Let $\mathfrak{g}$ and $\mathfrak{q}$ be Leibniz algebras. Then the following properties are equivalent:

a) $\mathfrak{g}$ and $\mathfrak{q}$ are Lie-isoclinic.

b) There exist a Leibniz algebra with trivial Lie-commutator $\mathfrak{a}$, a subalgebra $\mathfrak{h}$ of $\mathfrak{g} \times \mathfrak{a}$ with $\mathfrak{h}+Z_{\text {Lie }}(\mathfrak{g} \times \mathfrak{a})=\mathfrak{g} \times \mathfrak{a}$, and a two-sided ideal $\mathfrak{n}$ of $\mathfrak{h}$ with $\mathfrak{n} \cap[\mathfrak{h}, \mathfrak{h}]_{\text {Lie }}=0$, such that $\mathfrak{h} / \mathfrak{n}$ is isomorphic to $\mathfrak{q}$.

c) There exist a Leibniz algebra with trivial Lie-commutator $\mathfrak{b}$, a two-sided ideal $\mathfrak{m}$ of $\mathfrak{g} \times \mathfrak{b}$ with $\mathfrak{m} \cap[\mathfrak{g} \times \mathfrak{b}, \mathfrak{g} \times \mathfrak{b}]_{\text {Lie }}=0$, and a subalgebra $\mathfrak{i}$ of $\frac{\mathfrak{g} \times \mathfrak{b}}{\mathfrak{m}}$, with $\mathfrak{i}+Z_{\text {Lie }}\left(\frac{\mathfrak{g} \times \mathfrak{b}}{\mathfrak{m}}\right)=\frac{\mathfrak{g} \times \mathfrak{b}}{\mathfrak{m}}$, such that $\mathfrak{i}$ is isomorphic to $\mathfrak{q}$.

Proof. a) $\Longleftrightarrow$ b) Assume a), then by Theorem 3.19 there exists a Leibniz algebra with trivial Lie-commutator $\mathfrak{a}$, a Lie-central extension $\left(g^{\prime}\right)$, a Lie-isoclinic monomorphism (denoted by $\alpha$ ) from $\left(g^{\prime}\right)$ into $(g \times \mathfrak{a})$ and a Lie-isoclinic epimorphism (denoted by $\beta$ ) from $\left(g^{\prime}\right)$ onto $(q)$. Let $\mathfrak{h}=\operatorname{Im}(\alpha) \cong \mathfrak{g}^{\prime}$ and $\mathfrak{n}:=\operatorname{Ker}(\beta)$. Then $\mathfrak{h}+Z_{\text {Lie }}(\mathfrak{g} \times \mathfrak{a})=\mathfrak{g} \times \mathfrak{a}$ by Proposition 3.20, $\frac{\mathfrak{g}^{\prime}}{\mathfrak{n}} \cong \mathfrak{q}$ and we have by Proposition 3.11 that $\mathfrak{n} \cap[\mathfrak{h}, \mathfrak{h}]=0$.

Conversely, by letting $\mathfrak{g}^{\prime}=\mathfrak{h}$ then Proposition 3.20 provides a Lie-isoclinic monomorphism from $\left(g^{\prime}\right)$ into $(g \times \mathfrak{a})$ and a Lie-isoclinic epimorphism from $\left(g^{\prime}\right)$ onto $(q)$. The result follows by Theorem 3.19

a) $\Longleftrightarrow c$ ) Assume a), then by Theorem 3.19 there exists a Leibniz algebra with trivial Lie-commutator $\mathfrak{b}$, a central extension $\left(g^{\prime \prime}\right)$, a Lie-isoclinic epimorphism (denoted by $\alpha$ ) from $(g \times \mathfrak{b})$ onto $\left(g^{\prime \prime}\right)$, and a Lie-isoclinic monomorphism (denoted by $\beta$ ) from $(q)$ into $\left(g^{\prime \prime}\right)$. Let $\mathfrak{m}=\operatorname{Ker}(\alpha)$. Then $\frac{g \times \mathfrak{b}}{\mathfrak{m}} \cong \mathfrak{g}^{\prime \prime}$ and $\mathfrak{m} \cap[\mathfrak{g} \times \mathfrak{b}, \mathfrak{g} \times \mathfrak{b}]_{\text {Lie }}=0$ by Proposition 3.11. Also by Proposition 3.20 we have $\mathfrak{i}+Z_{\text {Lie }}\left(\mathfrak{g}^{\prime \prime}\right)=\mathfrak{g}^{\prime \prime}$, where $\mathfrak{i}=\operatorname{Im}(\beta) \cong \mathfrak{q}$.

Conversely, by letting $\mathfrak{g}^{\prime \prime}=\frac{g \times \mathfrak{b}}{\mathfrak{m}}$ we have by Proposition 3.20 that the embed$\operatorname{ding} \mathfrak{q} \cong \mathfrak{i}$ into $\mathfrak{g}^{\prime \prime}$ is a Lie-isoclinic monomorphism. Also Proposition 3.20 implies a Lie-isoclinic epimorphism from $\mathfrak{g} \times \mathfrak{b}$ to $\mathfrak{g}^{\prime \prime}$.

The result follows by Theorem 3.19 .

Remark 3.22 Let $\beta: \mathfrak{g} \rightarrow \mathfrak{q}$ be a Lie-isoclinic epimorphism. Then $\beta$ induces an isoclinism $(\eta, \xi): \mathfrak{g} \sim \mathfrak{q}$, where $\xi=\beta_{\mid[\mathfrak{g}, \mathfrak{g}]_{\text {Lie }}}$. Hence we have the following 
commutative diagram:

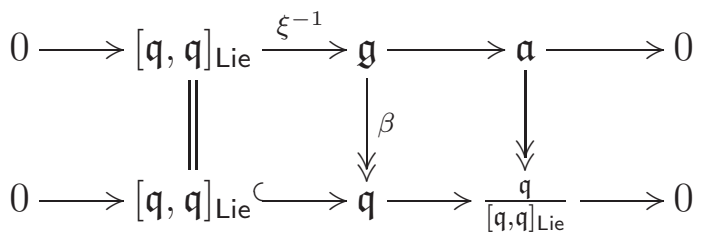

where $\mathfrak{a} \cong \frac{\mathfrak{g}}{[\mathfrak{g}, \mathfrak{g}]_{\llcorner i e}}$. On the other hand, if we fix $\mathfrak{q}$, while $\mathfrak{a}$ is a Leibniz algebra with trivial Lie-commutator and $\mathfrak{g}$ a Leibniz algebra which fits into (13) for some epimorphism $\beta$, then $[\mathfrak{g}, \mathfrak{g}]_{\text {Lie }}=\operatorname{Im}\left(\xi^{-1}\right)$ and $\operatorname{Ker}(\beta) \cap[\mathfrak{g}, \mathfrak{g}]_{\text {Lie }}=0$. Hence, diagram (13) determines all groups which map epi-isoclinic onto $\mathfrak{q}$.

\section{Lie-Isoclinism and the Schur Lie-multiplier}

In this section we analyze the connection between Lie-isoclinism and the second Lie-homology with trivial coefficients, which we call the Schur Lie-multiplier thanks to the isomorphism (1).

Consider the Lie-central extensions $(g): 0 \rightarrow \mathfrak{n} \stackrel{\chi}{\rightarrow} \mathfrak{g} \stackrel{\pi}{\rightarrow} \mathfrak{q} \rightarrow 0$ and $\left(g_{i}\right): 0 \rightarrow$ $\mathfrak{n}_{i} \stackrel{\chi_{i}}{\rightarrow} \mathfrak{g}_{i} \stackrel{\pi_{i}}{\rightarrow} \mathfrak{q}_{i} \rightarrow 0, i=1,2$. By Proposition 2 in [2], associated to $(g)$ there exists the following six-term exact sequence

$$
\mathfrak{n} \otimes \mathfrak{g}_{\text {Lie }} \longrightarrow \mathrm{HL}_{2}^{\text {Lie }}(\mathfrak{g}) \longrightarrow \mathrm{HL}_{2}^{\text {Lie }}(\mathfrak{q}) \stackrel{\theta(g)}{\longrightarrow} \mathfrak{n} \longrightarrow \mathrm{HL}_{1}^{\text {Lie }}(\mathfrak{g}) \longrightarrow \mathrm{HL}_{1}^{\text {Lie }}(\mathfrak{q}) \longrightarrow 0
$$

and from its proof easily follows that

$$
\operatorname{Im}(\theta(g))=\mathfrak{n} \cap[\mathfrak{g}, \mathfrak{g}]_{\text {Lie }} .
$$

Thus $\theta(g)$ induces a homomorphism $\theta^{\prime}(g): \mathrm{HL}_{2}^{\mathrm{Lie}}(\mathfrak{q}) \rightarrow[\mathfrak{g}, \mathfrak{g}]_{\text {Lie }}$ such that the sequence

$$
0 \rightarrow \operatorname{Ker}(\theta(g)) \rightarrow \mathrm{HL}_{2}^{\text {Lie }}(\mathfrak{q}) \stackrel{\theta^{\prime}(g)}{\rightarrow}[\mathfrak{g}, \mathfrak{g}]_{\text {Lie }} \stackrel{\pi^{\prime}}{\rightarrow}[\mathfrak{q}, \mathfrak{q}]_{\text {Lie }} \rightarrow 0
$$

is exact, where $\pi^{\prime}$ is induced by $\pi: \mathfrak{g} \rightarrow \mathfrak{q}$. The naturality of $\theta(g)$ with respect to homomorphisms of Lie-central extensions (see [6, Theorem 5.9]) yields the naturality of the sequence (16).

For the Lie-central extension $(g), \theta(g)$ is a monomorphism if and only if $\operatorname{Im}\left(\mathrm{HL}_{2}^{\mathrm{Lie}}(\pi)\right)=0$ by the exactness of (14), equivalently $\mathrm{HL}_{2}^{\mathrm{Lie}}(\pi)$ is the zero map, or equivalently, the sequence $0 \rightarrow \mathrm{HL}_{2}^{\text {Lie }}(\mathfrak{q}) \stackrel{\theta(g)}{\rightarrow} \mathfrak{n} \rightarrow \mathrm{HL}_{1}^{\text {Lie }}(\mathfrak{g}) \rightarrow \mathrm{HL}_{1}^{\text {Lie }}(\mathfrak{q}) \rightarrow 0$ is exact.

On the other hand, by (15) $\theta(g)$ is a monomorphism if and only if $\mathrm{HL}_{2}^{\text {Lie }}(\mathfrak{q}) \cong$ $\mathfrak{n} \cap[\mathfrak{g}, \mathfrak{g}]_{\text {Lie }}$. Having in mind [2, Proposition 3], the Lie-central extension $(g)$ with $\theta(g)$ a monomorphism is a Lie-trivial extension whenever $\mathrm{HL}_{2}^{\text {Lie }}(\mathfrak{q})=0$, so $\mathrm{HL}_{2}^{\mathrm{Lie}}(\mathfrak{q})$ measures the deficiency of a Lie-central extension to be a Lie-trivial extension. For this reason, we call a such Lie-central extension a quasi Lie-trivial extension. 
Lemma 4.1 Let $(\alpha, \beta, \gamma):\left(g_{1}\right) \rightarrow\left(g_{2}\right)$ be a homomorphism of Lie-central extensions and assume that $\gamma: \mathfrak{q}_{1} \rightarrow \mathfrak{q}_{2}$ is an isomorphism. Then the following statements are equivalent:

a) $(\alpha, \beta, \gamma)$ is a Lie-isoclinism.

b) $\mathrm{HL}_{2}^{\mathrm{Lie}}(\gamma)\left(\operatorname{Ker}\left(\theta\left(g_{1}\right)\right)\right)=\operatorname{Ker}\left(\theta\left(g_{2}\right)\right)$.

Proof. The naturality of (16) induces the following commutative diagram:

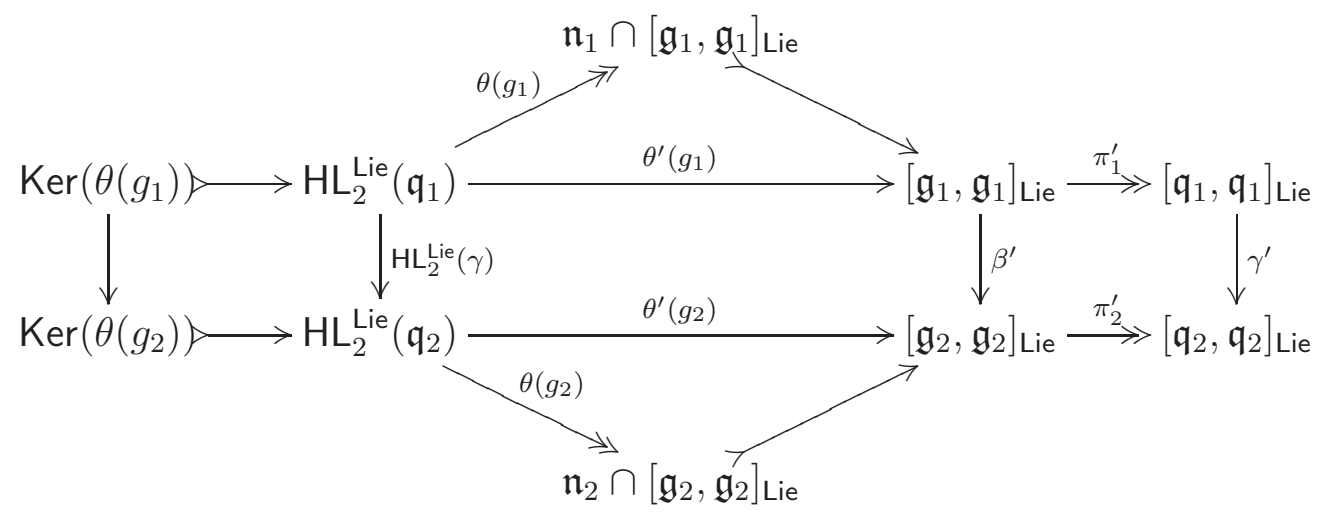

where $\theta^{\prime}\left(g_{i}\right), i=1,2$, are defined as above, $\beta^{\prime}=\beta_{\left[\left[\mathfrak{g}_{1}, \mathfrak{g}_{1}\right]_{\text {Lie }}\right.}, \gamma^{\prime}=\gamma_{\left[\left[\mathfrak{q}_{1}, \mathfrak{q}_{1}\right]_{\text {Lie }}\right.}$.

Since $\gamma$ is a isomorphism, then $\gamma^{\prime}$ and $\operatorname{HL}_{2}^{\text {Lie }}(\gamma)$ are also isomorphisms, the restriction of $\mathrm{HL}_{2}^{\text {Lie }}(\gamma)$ to $\operatorname{Ker}\left(\theta\left(g_{1}\right)\right)$ is a monomorphism and $\beta$ is an epimorphism.

By the commutativity of the left hand square in the diagram, we have that $\mathrm{HL}_{2}^{\text {Lie }}(\gamma)\left(\operatorname{Ker}\left(\theta\left(g_{1}\right)\right)\right) \subseteq \operatorname{Ker}\left(\theta\left(g_{2}\right)\right)$. Conversely, for any $y \in \operatorname{Ker}\left(\theta\left(g_{2}\right)\right)$, there exists $x \in \mathrm{HL}_{2}^{\text {Lie }}\left(\mathfrak{q}_{1}\right)$ such that $\mathrm{HL}_{2}^{\text {Lie }}(\gamma)(x)=y$. Now $0=\theta^{\prime}\left(g_{2}\right)(y)=\theta^{\prime}\left(g_{2}\right) \circ$ $\mathrm{HL}_{2}^{\text {Lie }}(\gamma)(x)=\beta_{\mid}^{\prime} \circ \theta^{\prime}\left(g_{1}\right)(x)$. Hence $x \in \operatorname{Ker}\left(\theta\left(g_{1}\right)\right)$ whenever $\beta_{\mid}^{\prime}$ is a monomorphism.

Consequently, statement $b$ ) holds if and only if $\beta_{\mid}^{\prime}$ is a monomorphism if and only if $(\alpha, \beta, \gamma)$ is a Lie-isoclinism (having in mind Proposition 3.10).

Lemma 4.2 Let $\eta: \mathfrak{q}_{1} \rightarrow \mathfrak{q}_{2}$ be an isomorphism and $(\widetilde{g})=\left(g_{1} \times g_{2}^{\eta}\right)^{\Delta}$ as in section 目, then

$$
\operatorname{Ker}(\theta(\widetilde{g}))=\mathrm{HL}_{2}^{\text {Lie }}(\eta)^{-1}\left(\operatorname{Ker}\left(\theta\left(g_{2}\right)\right)\right) \cap \operatorname{Ker}\left(\theta\left(g_{1}\right)\right) .
$$

Proof. From diagram (4) and the naturality of $\theta(g)$ we have that

$$
\theta(\widetilde{g})=\theta\left(g_{1}\right) \times \theta\left(g_{2}\right) \circ \mathrm{HL}_{2}^{\mathrm{Lie}}\left(\gamma_{2}\right) .
$$

Since $\mathrm{HL}_{2}^{\mathrm{Lie}}\left(\gamma_{2}\right)$ is an isomorphism, then $\operatorname{Ker}\left(\theta\left(g_{2}\right) \circ \mathrm{HL}_{2}^{\text {Lie }}\left(\gamma_{2}\right)\right)=\mathrm{HL}_{2}^{\mathrm{Lie}}\left(\gamma_{2}\right)^{-1}\left(\operatorname{Ker}\left(\theta\left(g_{2}\right)\right)\right)$, hence the required equality follows.

Theorem 4.3 Let $\eta: \mathfrak{q}_{1} \rightarrow \mathfrak{q}_{2}$ be an isomorphism. Then the following statements are equivalent: 
a) $\eta$ induces a Lie-isoclinism from $\left(g_{1}\right)$ to $\left(g_{2}\right)$.

b) There exists an isomorphism $\beta^{\prime}:\left[\mathfrak{g}_{1}, \mathfrak{g}_{1}\right]_{\text {Lie }} \rightarrow\left[\mathfrak{g}_{2}, \mathfrak{g}_{2}\right]_{\text {Lie }}$ with $\beta^{\prime} \circ \theta^{\prime}\left(g_{1}\right)=$ $\theta^{\prime}\left(g_{2}\right) \circ \mathrm{HL}_{2}^{\mathrm{Lie}}(\eta)$.

c) $\mathrm{HL}_{2}^{\mathrm{Lie}}(\eta)\left(\operatorname{Ker}\left(\theta\left(g_{1}\right)\right)\right)=\operatorname{Ker}\left(\theta\left(g_{2}\right)\right)$.

Proof. a) $\Rightarrow b)$ Let $\left(\eta, \beta^{\prime}\right):\left(g_{1}\right) \sim\left(g_{2}\right)$ be. Then Propositions 3.14 and 3.10 imply that $\beta^{\prime}=\tau_{2}^{\prime} \circ \tau_{1}^{\prime-1}$, where $\tau_{i}^{\prime}=\tau_{i \mid\left[\mathfrak{g}_{\mathrm{g}, \mathfrak{g}} L_{\text {Lie }}\right.}$ and the proof of Proposition 3.14 shows that both of them are isomorphisms.

Then the naturality of sequence (16) applied to diagrams (44) implies

$$
\theta^{\prime}\left(g_{2}\right) \circ \mathrm{HL}_{2}^{\mathrm{Lie}}\left(\gamma_{2}\right)=\tau_{2}^{\prime} \circ \theta^{\prime}(\widetilde{\mathfrak{g}})=\tau_{2}^{\prime} \circ \tau_{1}^{\prime-1} \circ \theta^{\prime}\left(g_{1}\right) \circ \mathrm{HL}_{2}^{\mathrm{Lie}}\left(\gamma_{1}\right),
$$

hence the required equality.

b) $\Rightarrow c)$ Since $\beta^{\prime} \circ \theta^{\prime}\left(g_{1}\right)=\theta^{\prime}\left(g_{2}\right) \circ \mathrm{HL}_{2}^{\text {Lie }}(\eta)$, then $\mathrm{HL}_{2}^{\text {Lie }}(\eta)\left(\operatorname{Ker}\left(\theta\left(g_{1}\right)\right)\right) \subseteq$ $\operatorname{Ker}\left(\theta\left(g_{2}\right)\right)$. The converse inclusion is followed thanks to be $\beta^{\prime}$ an isomorphism.

$c) \Rightarrow a$ ) Let $(\widetilde{g})$ be as above, then Lemma 4.2 implies that

$$
\operatorname{Ker}(\theta(\widetilde{g}))=\mathrm{HL}_{2}^{\text {Lie }}(\eta)^{-1} \circ \mathrm{HL}_{2}^{\text {Lie }}(\eta)\left(\operatorname{Ker}\left(\theta\left(g_{1}\right)\right)\right) \cap \operatorname{Ker}\left(\theta\left(g_{1}\right)\right)=\operatorname{Ker}\left(\theta\left(g_{1}\right)\right) .
$$

From diagram (4), the epimorphisms $\left(\sigma_{i}, \tau_{i}, \gamma_{i}\right):(\widetilde{g}) \sim\left(g_{i}\right)$ and the equality (17) imply that

$$
\mathrm{HL}_{2}^{\mathrm{Lie}}\left(\gamma_{i}\right)(\operatorname{Ker}(\theta(\widetilde{g})))=\operatorname{Ker}\left(\theta\left(g_{i}\right)\right), i=1,2 .
$$

Lemma 4.1 implies that $\left(\sigma_{i}, \tau_{i}, \gamma_{i}\right):(\widetilde{g}) \sim\left(g_{i}\right), i=1,2$. Now Proposition 3.14 ends the proof.

\section{Corollary 4.4}

a) Let Autcl $(g)$ be the group of Lie-autoclinisms of $(g)$. Then

$$
\operatorname{Autcl}(g) \cong\left\{\eta \mid \eta \in \operatorname{Aut}(\mathfrak{q}) \text {, such that } \mathrm{HL}_{2}^{\text {Lie }}(\operatorname{Ker}(\theta(g)))=\operatorname{Ker}(\theta(g))\right\} .
$$

b) If $(g)$ is a quasi Lie-trivial extension, then $\operatorname{Autcl}(g) \cong \operatorname{Aut}(\mathfrak{q})$.

c) Any two quasi Lie-trivial extensions of a given Leibniz algebra are Lieisoclinic.

Proof. Direct application of Theorem 4.3 .

Following [2], a Lie-central extension $0 \rightarrow \mathfrak{n} \stackrel{\chi}{\rightarrow} \mathfrak{g} \stackrel{\pi}{\rightarrow} \mathfrak{q} \rightarrow 0$ is called a Liestem extension if $\mathfrak{g}_{\text {Lie }} \cong \mathfrak{q}_{\text {Lie }}$. A Lie-stem cover is a Lie-stem extension such that the induced map $H L_{2}^{\text {Lie }}(\mathfrak{g}) \rightarrow H L_{2}^{\text {Lie }}(\mathfrak{q})$ is the zero map. Proposition 6 in [2] characterizes Lie-stem covers by the fact that the induced map $\theta: H L_{2}^{\text {Lie }}(\mathfrak{q}) \rightarrow \mathfrak{n}$ is an isomorphism. 
Consider $\mathfrak{q}$ a Leibniz algebra for which there exists a free presentation $0 \rightarrow$ $\mathfrak{s} \rightarrow \mathfrak{f} \stackrel{\tau}{\rightarrow} \mathfrak{q} \rightarrow 0$ satisfying $\mathfrak{s} \subseteq[\mathfrak{f}, \mathfrak{f}]_{\text {Lie }}$. For instance $\mathfrak{q}$ is a free Leibniz algebra, then $\mathfrak{f} \cong \mathfrak{q}, \mathfrak{s}=0$. Under this assumption, it is easy to check that the map $H L_{2}^{\text {Lie }}(\mathfrak{q}) \cong \frac{\mathfrak{s} \cap[f, f] \text { Lie }}{[\mathfrak{s}, f] \text { Lie }} \rightarrow \frac{\mathfrak{s}}{[\mathfrak{s}, f] \text { Lie }}$ is an epimorphism, so Proposition 5 (c) and Example 2 (b) in [2] provides the following Lie-stem cover:

$$
0 \rightarrow \frac{\mathfrak{s}}{[\mathfrak{s}, \mathfrak{f}]_{\text {Lie }}} \rightarrow \frac{\mathfrak{f}}{[\mathfrak{s}, \mathfrak{f}]_{\text {Lie }}} \stackrel{\bar{\tau}}{\rightarrow} \mathfrak{q} \rightarrow 0
$$

The above remark shows that there exists at least one Lie-stem cover associated to a Leibniz algebra having a free presentation $0 \rightarrow \mathfrak{s} \rightarrow \mathfrak{f} \stackrel{\tau}{\rightarrow} \mathfrak{q} \rightarrow 0$ satisfying $\mathfrak{s} \subseteq[\mathfrak{f}, \mathfrak{f}]_{\text {Lie }}$.

Corollary 4.5 All Lie-stem covers of a given Leibniz algebra $\mathfrak{q}$ having a free presentation $0 \rightarrow \mathfrak{s} \rightarrow \mathfrak{f} \stackrel{\tau}{\rightarrow} \mathfrak{q} \rightarrow 0$ satisfying $\mathfrak{s} \subseteq[\mathfrak{f}, \mathfrak{f}]_{\text {Lie }}$ are mutually Lie-isoclinic.

Proof. For two Lie-stem covers $\left(g_{1}\right)$ and $\left(g_{2}\right)$ of $\mathfrak{q}, \theta\left(g_{1}\right)$ and $\theta\left(g_{2}\right)$ are isomorphisms by [2, Proposition 6], then $\operatorname{Ker}\left(\theta\left(g_{1}\right)\right)=\operatorname{Ker}\left(\theta\left(g_{2}\right)\right)$ and Proposition 4.3 ends the proof.

\section{Acknowledgements}

Second author was supported by Ministerio de Economía y Competitividad (Spain) (European FEDER support included), grant MTM2013-43687-P.

\section{References}

[1] J. M. Casas, M. A. Insua, M. Ladra and S. Ladra: An algorithm for the classification of 3-dimensional complex Leibniz algebras, Linear Algebra Appl. 436 (9) (2012), 3747-3756.

[2] J. M. Casas and E. Khmaladze: On Lie-central extensions of Leibniz algebras, RACSAM (2016), DOI 10.1007/s13398-016-0274-6.

[3] J. M. Casas and T. Van der Linden: A relative theory of universal central extensions, Pré-Publicaçoes do Departamento de Matemática, Universidade de Coimbra Preprint Number 09- (2009).

[4] J. M. Casas and T. Van der Linden: Universal central extensions in semiabelian categories, Appl. Categor. Struct. 22 (1) (2014), 253-268.

[5] C. Cuvier: Algèbres de Leibnitz: définitions, propriétés, Ann. Sci. Écol. Norm. Sup. 27 (4) (1994), 1-45. 
[6] T. Everaert and T. Van der Linden: Baer invariants in semi-abelian categories I: General theory, Theory Appl. Categ. 12 (1) (2004), 1-33.

[7] P. Hall: The classification of prime-power groups, J. Reine Angew. Math. 182 (1940), 130-141.

[8] G. Janelidze and G. M. Kelly: Galois theory and a general notion of central extension, J. Pure Appl. Algebra 97 (1994), 135-161.

[9] G. Janelidze, L. Márki and W. Tholen: Semi-abelian categories, J. Pure Appl. Algebra 168 (2002), 367-386.

[10] J.-L. Loday: Cyclic homology, Grundl. Math. Wiss. Bd. 301, Springer (1992).

[11] J.-L. Loday: Une version non commutative des algèbres de Lie: les algèbres de Leibniz, L'Enseignement Mathématique 39 (1993), 269-292.

[12] M. R. R. Moghaddam and F. Parvaneh: On the isoclinism of a pair of Lie algebras and factor sets, Asian-Eur. J. Math. 2 (2) (2009), 213-225.

[13] H. Mohammadzadeh, A. R. Salemkar and Z. Riyahi: Isoclinic extensions of Lie algebras, Turkish J. Math. 37 (4) (2013), 598-606.

[14] K. Moneyhun: Isoclinisms in Lie algebras, Algebras Groups Geom. 11 (1) (1994), 9-22.

[15] A. R. Salemkar, V. Alamian and H. Mohammadzadeh: Some properties of the Schur multiplier and covers of Lie algebras, Comm. Algebra 36 (2) (2008), 697-707.

[16] A. R. Salemkar, H. Bigdely and V. Alamian: Some properties on isoclinism of Lie algebras and covers, J. Algebra Appl. 7 (4) (2008), 507-516.

[17] S. Sheikh-Mohseni, F. Saeedi and M. Badrkhani Asl: On special subalgebras of derivations of Lie algebras, Asian-Eur. J. Math. 8 (2) (2015), 1550032, 12 pp. 